\title{
Quadratic Differentials and Equivariant Deformation Theory of Curves
}

\author{
B. Köck and A. Kontogeorgis
}

November 12, 2018

\begin{abstract}
Given a finite $p$-group $G$ acting on a smooth projective curve $X$ over an algebraically closed field $k$ of characteristic $p$, the dimension of the tangent space of the associated equivariant deformation functor is equal to the dimension of the space of coinvariants of $G$ acting on the space $V$ of global holomorphic quadratic differentials on $X$. We apply known results about the Galois module structure of RiemannRoch spaces to compute this dimension when $G$ is cyclic or when the action of $G$ on $X$ is weakly ramified. Moreover we determine certain subrepresentations of $V$, called $p$-rank representations.
\end{abstract}

\section{Introduction}

Let $X$ be a non-singular complete curve of genus $g_{X} \geq 2$ defined over an algebraically closed field $k$ of characteristic $p$ and let $G$ be a subgroup of the automorphism group of $X$. The equivariant deformation problem associated with this situation is to determine in how many ways $X$ can be deformed to another curve that also allows $G$ as a group of automorphisms. More precisely, we are led to study the deformation functor $D$ which maps any local Artinian $k$-algebra $A$ to the set of isomorphism classes of lifts of $(G, X)$ to an action of $G$ on a smooth scheme $\tilde{X}$ over $\operatorname{Spec}(A)$, see [1 for a detailed description of $D$. The dimension of the tangent space of $D$ (in the sense of Schlessinger [16]) can be thought of as a (crude) answer to the abovementioned equivariant deformation problem. In [1], Bertin and Mézard have shown that the tangent space of $D$ is isomorphic to the equivariant cohomology $H^{1}\left(G, \mathcal{T}_{X}\right)$ of $(G, X)$ with values in the tangent sheaf $\mathcal{T}_{X}$ of $X$. A more 
detailed analysis of the above-mentioned equivariant deformation problem also involves determining obstructions in $H^{2}\left(G, \mathcal{T}_{X}\right)$ and studying the structure of the versal deformation ring associated with $D$, see [1] and [4].

In the classical case, i.e. when $k=\mathbb{C}$ (see e.g. [6, Section V.2.2]) or, more generally, when the action of $G$ on $X$ is tame (see [1, p. 206] and [10, formula (40)]), the dimension of the tangent space of $D$ is known to be equal to $3 g_{Y}-3+r$ where $g_{Y}$ denotes the genus of the quotient curve $Y=X / G$ and $r$ denotes the cardinality of the branch locus $Y_{\text {ram }}$; note that $3 g_{Y}-3$ is the dimension of the moduli space of curves of genus $g_{Y}$ and each branch point adds one further degree of freedom in our deformation problem, as expected.

In the case of wild ramification, the computation of the dimension of the tangent space of $D$ turns out to be a difficult problem. In this paper, we disregard the known case of tame ramification and assume that the characteristic $p$ of $k$ is positive and that $G$ is a $p$-group.

Formulas for the dimension of $H^{1}\left(G, \mathcal{T}_{X}\right)$ can be derived from results in [1] and [4] when in addition $G$ is a cyclic group or $X$ is an ordinary curve (or, more generally, the action of $G$ on $X$ is weakly ramified), see Remarks 2.4 and 3.2. Furthermore, when $G$ is an elementary abelian group, the secondnamed author computes this dimension in [10] using the Lyndon-HochschildSerre spectral sequence.

The object of this paper is to pursue the following alternative method for computing the dimension of $H^{1}\left(G, \mathcal{T}_{X}\right)$. In [11], the second-named author has shown that this dimension is equal to the dimension of the space $H^{0}\left(X, \Omega_{X}^{\otimes 2}\right)_{G}$ of coinvariants of $G$ acting on the space of global holomorphic quadratic differentials on $X$. We will use known results on the Galois module structure of Riemann-Roch spaces $H^{0}\left(X, \mathcal{O}_{X}(D)\right)$ for certain $G$-invariant divisors $D$ on $X$ to determine the dimension of $H^{0}\left(X, \Omega_{X}^{\otimes 2}\right)_{G}$.

We now fix some notations. Let $P_{1}, \ldots, P_{r}$ denote a complete set of representatives for the $G$-orbits of ramified points. The corresponding decomposition groups, ramification indices and coefficients of the ramification divisor are denoted by $G\left(P_{j}\right), e_{0}\left(P_{j}\right)$ and $d\left(P_{j}\right), j=1, \ldots, r$, respectively.

Section 2 of this paper will deal with the case when $G$ is cyclic. Generalizing a result of Nakajima [14, Theorem 1] from the case when $G$ is cyclic of order $p$ to the case when $G$ is an arbitrary cyclic $p$-group, Borne [3, Theorem 7.23] has explicitly determined the Galois module structure of $H^{0}\left(X, \mathcal{O}_{X}(D)\right)$ for any $G$-invariant divisor $D$ on $X$ of degree greater than $2 g_{X}-2$. Although the formulation of Borne's theorem requires quite involved definitions and is in particular difficult to state, its consequence for the dimension of $H^{0}\left(X, \Omega_{X}^{\otimes 2}\right)_{G}$ 
is simple:

$$
\operatorname{dim}_{k} H^{0}\left(X, \Omega_{X}^{\otimes 2}\right)_{G}=3 g_{Y}-3+\sum_{j=1}^{r}\left\lfloor\frac{2 d\left(P_{j}\right)}{e_{0}\left(P_{j}\right)}\right\rfloor,
$$

see Corollary 2.3. This result can also be derived from core results in [1] and becomes [1, Proposition 4.1.1] if $G$ is cyclic.

In Section 3 we assume that the action of $G$ on $X$ is weakly ramified, i.e. that the second ramification group $G_{2}(P)$ vanishes for all $P \in X$, and prove the formula

$$
\operatorname{dim}_{k} H^{0}\left(X, \Omega_{X}^{\otimes 2}\right)_{G}=3 g_{Y}-3+\sum_{j=1}^{r} \log _{p}\left|G\left(P_{j}\right)\right|+ \begin{cases}2 r & \text { if } p>3 \\ r & \text { if } p=2 \text { or } 3\end{cases}
$$

see Theorem 3.1. This formula matches up with results of Cornelissen and Kato [4, Theorem 4.5 and Theorem 5.1(b)] for the deformation of ordinary curves, see Remark 3.2. The proof of our result proceeds along the following lines. Let $W$ denote the space of global meromorphic quadratic differentials on $X$ which may have a pole of order at most 3 at each ramified point and which are holomorphic everywhere else. A result of the first-named author [9, Theorem 4.5] implies that $W$ is a free $k[G]$-module and that its rank and hence the dimension of $W_{G}$ is equal to $3\left(g_{Y}-1+r\right)$, see Proposition 3.4. The difference between this dimension and the dimension of $H^{0}\left(X, \Omega_{X}^{\otimes 2}\right)_{G}$ can be expressed in terms of group homology of $G$ acting on the space of global sections of a certain skyscraper sheaf and can be computed using a spectral sequence argument, see Proposition 3.6 and Lemma 3.7.

In Section 4 we first show that there exists an effective $G$-invariant canonical divisor $D$ on $X$, see Lemma 4.2. This allows us to split the $k[G]$-module $H^{0}\left(X, \Omega_{X}^{\otimes 2}\right) \cong H^{0}\left(X, \Omega_{X}(D)\right)$ into its semisimple and nilpotent part with respect to the corresponding Cartier operator [20]:

$$
H^{0}\left(X, \Omega_{X}(D)\right) \cong H_{D}^{\mathrm{s}} \oplus H_{D}^{\mathrm{n}} .
$$

Little seems to be known about the $k[G]$-module structure of the nilpotent part $H_{D}^{\mathrm{n}}$. We use a result of Nakajima [13, Theorem 1] to show that the semisimple part $H_{D}^{\mathrm{s}}$ is a free $k[G]$-module and to compute its rank and hence the dimension of $\left(H_{D}^{\mathrm{s}}\right)_{G}$ provided $D$ satisfies further conditions, see Theorem 4.3. In cases the $k[G]$-module structure of $H^{0}\left(X, \Omega_{X}^{\otimes 2}\right)$ is known (such as Section 2) we then also get information about $H_{D}^{\mathrm{n}}$, see Corollary 4.5.

Section 5 is an appendix and gives an account of a structure theorem (Theorem 5.2) for weakly ramified Galois extensions of local fields. This structure theorem is used in Section 3, but only in the case $p=2$. We finally derive a 
feature of the Weierstrass semigroup at any ramified point of $X$ if $p=2$ and the action of $G$ on $X$ is weakly ramified, see Corollary 5.3.

Acknowledgments: The second-named author would like to thank Gunther Cornelissen and Michel Matignon for pointing him to the work of Niels Borne, Bernhard Köck and Nicolas Stalder.

Notations. Throughout this paper $X$ is a connected smooth projective curve over an algebraically closed field $k$ of positive characteristic $p$ and $G$ is a finite subgroup of the automorphism group of $X$ whose order is a power of $p$. We also assume that the genus $g_{X}$ of $X$ is at least 2. For any point $P \in X$ let $G(P)=\{g \in G: g(P)=P\}$ denote the decomposition group and $e_{0}(P)=|G(P)|$ the ramification index at $P$. The $i^{\text {th }}$ ramification group at $P$ in lower notation is

$$
G_{i}(P)=\left\{g \in G(P): g\left(t_{P}\right)-t_{P} \in\left(t_{P}^{i+1}\right)\right\}
$$

where $t_{P}$ is a local parameter at $P$. Let $e_{i}(P)$ denote its order. Note that $e_{0}(P)=e_{1}(P)$ because $G$ is a $p$-group. Let

$$
\pi: X \rightarrow Y:=X / G
$$

denote the canonical projection, and let $g_{Y}$ denote the genus of $Y$. We use the notation $X_{\text {ram }}$ for the set of ramification points and $Y_{\text {ram }}=\pi\left(X_{\text {ram }}\right)$ for the set of branch points of $\pi$. We fix a complete set $P_{1}, \ldots, P_{r}$ of representatives for the $G$-orbits of ramified points in $X$; in particular we have $r=\left|Y_{\text {ram }}\right|$. The sheaves of differentials and of relative differentials on $X$ are denoted by $\Omega_{X}$ and $\Omega_{X / Y}$, respectively. Furthermore for any divisor $D$ on $X$ we use the notation $\Omega_{X}(D)$ for the sheaf $\Omega_{X} \otimes_{\mathcal{O}_{X}} \mathcal{O}_{X}(D)$. If $D=\sum_{P \in X} n_{P}[P]$ is an effective divisor, we write

$$
D_{\text {red }}:=\sum_{P \in X: n_{P} \neq 0}[P]
$$

for the associated reduced divisor. Let

$$
R=\sum_{P \in X} d(P)[P]
$$

denote the ramification divisor of $\pi$; then $R_{\text {red }}=\sum_{P \in X_{\text {ram }}}[P]$ is the reduced ramification divisor. Note that

$$
d(P)=\sum_{i=0}^{\infty}\left(e_{i}(P)-1\right)
$$

by Hilbert's different formula [21, Theorem 3.8.7]. The notation $K_{Y}$ stands for a canonical divisor on $Y$; the divisor $K_{X}:=\pi^{*} K_{Y}+R$ is then a $G$ invariant canonical divisor on $X$ by [7, Proposition IV 2.3]. For any real number $x$, the notation $\lfloor x\rfloor$ means the largest integer less than or equal to $x$. 


\section{The Cyclic Case}

In this section we assume that the group $G$ is cyclic of order $p^{\nu}$ and compute the dimension $\operatorname{dim}_{k} H^{1}\left(G, \mathcal{T}_{X}\right)=\operatorname{dim}_{k} H^{0}\left(X, \Omega_{X}^{\otimes 2}\right)_{G}$ of the tangent space of the deformation functor associated with $G$ acting on $X$, see Corollary 2.3. We derive Corollary 2.3 from Theorem 2.1 which in turn is a consequence of a theorem of Borne [3, Theorem 7.23]. While a lot of notations and explanations are needed to formulate Borne's very fine statement, Theorem 2.1 is easy to state and sufficient to derive the important Corollary 2.3. A different way of formulating Corollary 2.3 is given in Lemma 2.5 and a different way of proving it is outlined in Remark 2.4.

Let $\sigma$ denote a generator of $G$. Let $V$ denote the $k[G]$-module with $k$-basis $e_{1}, \ldots, e_{p^{\nu}}$ and $G$-action given by $\sigma\left(e_{1}\right)=e_{1}$ and $\sigma\left(e_{l}\right)=e_{l}+e_{l-1}$ for $l=$ $2, \ldots, p^{\nu}$. It is well-known that the submodules $V_{l}:=\operatorname{Span}_{k}\left(e_{1}, \ldots, e_{l}\right), l=$ $1, \ldots, p^{\nu}$, of $V$ form a set of representatives for the set of isomorphism classes of indecomposable $k[G]$-modules. In particular for every finitely generated $k[G]$-module $M$ there are some integers $m_{l}(M) \geq 0, l=1, \ldots, p^{\nu}$, such that

$$
M \cong \bigoplus_{l=1}^{p^{\nu}} V_{l}^{\oplus m_{l}(M)}
$$

let $\operatorname{Tot}(M)=\sum_{l=1}^{p^{\nu}} m_{l}(M)$ denote the total number of direct summands.

Theorem 2.1. Let $D=\sum_{P \in X} n_{P}[P]$ be a $G$-invariant divisor on $X$ such that $\operatorname{deg}(D)>2 g_{X}-2$. Then we have

$$
\operatorname{Tot}\left(H^{0}\left(X, \mathcal{O}_{X}(D)\right)\right)=1-g_{Y}+\sum_{Q \in Y}\left\lfloor\frac{n_{\tilde{Q}}}{e_{0}(\tilde{Q})}\right\rfloor
$$

where $\tilde{Q}$ denotes any point in the fiber $\pi^{-1}(Q)$.

Remark 2.2. Our global assumption that $g_{X} \geq 2$ is not necessary for this theorem.

Proof. Borne defines certain divisors $D(l), l=1, \ldots, p^{\nu}$, on $Y$ and proves that

$$
m_{l}\left(H^{0}\left(X, \mathcal{O}_{X}(D)\right)\right)=\operatorname{deg}(D(l))-\operatorname{deg}(D(l+1)) \quad \text { for } l=1, \ldots, p^{\nu}-1
$$

and

$$
m_{p^{\nu}}\left(H^{0}\left(X, \mathcal{O}_{X}(D)\right)\right)=1-g_{Y}+\operatorname{deg}\left(D\left(p^{\nu}\right)\right)
$$


see [3, Theorem 7.23]. (The reader who wants to match this with [3] may find useful to observe that $(p-1)+(p-1) p+\ldots+(p-1) p^{\nu-1}$ is the $p$-adic expansion of $p^{\nu}-1$.) We therefore have

$$
\operatorname{Tot}\left(H^{0}\left(X, \mathcal{O}_{X}(D)\right)\right)=\sum_{l=1}^{p^{\nu}} m_{l}\left(H^{0}\left(X, \mathcal{O}_{X}(D)\right)\right)=\operatorname{deg}(D(1))+1-g_{Y}
$$

We now explain the definition of $D(1)$. For each $\mu=0, \ldots, \nu$ let $H_{\mu}$ denote the unique subgroup of $G$ of order $p^{\mu}$ and set $X_{\mu}:=X / H_{\mu}$. Furthermore let $\pi_{\mu}: X_{\mu-1} \rightarrow X_{\mu}$ denote the canonical projection. For each divisor $E$ on $X_{\mu-1}$ set $\left(\pi_{\mu}\right)_{*}^{0}(E):=\left\lfloor\frac{1}{p}\left(\pi_{\mu}\right)_{*}(E)\right\rfloor$ where $\lfloor\cdot\rfloor$ denotes the integral part of a divisor, taken coefficient by coefficient. Then $D(1)$ is defined as

$$
D(1)=\left(\pi_{\nu}\right)_{*}^{0} \ldots\left(\pi_{1}\right)_{*}^{0}(D),
$$

see [3, Definition 7.22 and Theorem 7.23]. Now Theorem 2.1] follows from the formula

$$
D(1)=\sum_{Q \in Y}\left\lfloor\frac{n_{\tilde{Q}}}{e_{0}(\tilde{Q})}\right\rfloor[Q] .
$$

We prove this formula by induction on $\nu$. It is obvious if $\nu=0$. So let $\nu \geq 1$. By the inductive hypothesis we have

$$
\left(\pi_{\nu-1}\right)_{*}^{0} \ldots\left(\pi_{1}\right)_{*}^{0}(D)=\sum_{R \in X_{\nu-1}}\left\lfloor\frac{n_{\tilde{R}}}{\left|G_{0}(\tilde{R}) \cap H_{\nu-1}\right|}\right\rfloor[R]
$$

where, as above, $\tilde{R}$ denotes any point in the fiber $\left(\pi_{\nu-1} \circ \ldots \circ \pi_{1}\right)^{-1}(R)$. Thus, if $Q \in Y$ is not a branch point of $\pi_{\nu}$, the coefficient of the divisor $D(1)$ at $Q$ is equal to

$$
\left\lfloor\frac{n_{\tilde{Q}}}{\left|G_{0}(\tilde{Q}) \cap H_{\nu-1}\right|}\right\rfloor=\left\lfloor\frac{n_{\tilde{Q}}}{\left|G_{0}(\tilde{Q})\right|}\right\rfloor=\left\lfloor\frac{n_{\tilde{Q}}}{e_{0}(\tilde{Q})}\right\rfloor,
$$

as desired. Otherwise we have $\left|G_{0}(\tilde{Q}) \cap H_{\nu-1}\right|=\left|H_{\nu-1}\right|=p^{\nu-1}$ and the coefficient of $D(1)$ at $Q$ is equal to

$$
\left\lfloor\frac{\left\lfloor\frac{n_{\tilde{Q}}}{p^{\nu-1}}\right\rfloor}{p}\right\rfloor \stackrel{(*)}{=}\left\lfloor\frac{n_{\tilde{Q}}}{p^{\nu}}\right\rfloor=\left\lfloor\frac{n_{\tilde{Q}}}{e_{0}(\tilde{Q})}\right\rfloor
$$

again as stated. (To see $(*)$ write $n_{\tilde{Q}}$ in the form $a p^{\nu}+b p^{\nu-1}+c$ with some $a \geq 0, b \in\{0, \ldots, p-1\}$ and $c \in\left\{0, \ldots, p^{\nu-1}\right\}$.) 
Corollary 2.3. We have

$$
\operatorname{dim}_{k} H^{0}\left(X, \Omega_{X}^{\otimes 2}\right)_{G}=3 g_{Y}-3+\sum_{j=1}^{r}\left\lfloor\frac{2 d\left(P_{j}\right)}{e_{0}\left(P_{j}\right)}\right\rfloor .
$$

Proof. Let $K_{Y}=\sum_{Q \in Y} m_{Q}[Q]$ be a canonical divisor on $Y$ and let $K_{X}$ denote the canonical $G$-invariant divisor $\pi^{*} K_{Y}+R$ on $X$. As $\operatorname{dim}\left(V_{l}\right)_{G}=1$ for all $l=1, \ldots, p^{\nu}$ we have

$$
\begin{aligned}
& \operatorname{dim}_{k} H^{0}\left(X, \Omega_{X}^{\otimes 2}\right)_{G}=\operatorname{Tot}\left(H^{0}\left(X, \Omega_{X}^{\otimes 2}\right)\right) \\
&=\operatorname{Tot}\left(H^{0}\left(X, \mathcal{O}_{X}\left(2 K_{X}\right)\right)\right)=\operatorname{Tot}\left(H^{0}\left(X, \mathcal{O}_{X}\left(2 \pi^{*} K_{Y}+2 R\right)\right)\right) .
\end{aligned}
$$

For any $Q \in Y$ the coefficient of the divisor $2 \pi^{*} K_{Y}+2 R$ at any point $\tilde{Q} \in \pi^{-1}(Q)$ is $2 e_{0}(\tilde{Q}) m_{Q}+2 d(\tilde{Q})$. Thus from Theorem 2.1 we obtain

$$
\begin{aligned}
& \operatorname{dim}_{k} H^{0}\left(X, \Omega_{X}^{\otimes 2}\right)_{G} \\
& =1-g_{Y}+2\left(\sum_{Q \in Y} m_{Q}\right)+\sum_{Q \in Y}\left\lfloor\frac{2 d(\tilde{Q})}{e_{0}(\tilde{Q})}\right\rfloor \\
& =3 g_{Y}-3+\sum_{j=1}^{r}\left\lfloor\frac{2 d\left(P_{j}\right)}{e_{0}\left(P_{j}\right)}\right\rfloor,
\end{aligned}
$$

as stated.

Remark 2.4. The following chain of equalities sketches a different approach to prove Corollary 2.3 based on core results of the paper [1] by Bertin and Mézard.

$$
\begin{aligned}
& \operatorname{dim}_{k} H^{0}\left(X, \Omega_{X}^{\otimes 2}\right)_{G} \\
& =\operatorname{dim}_{k} H^{1}\left(G, \mathcal{T}_{X}\right) \\
& =\operatorname{dim}_{k} H^{1}\left(Y, \pi_{*}^{G}\left(\mathcal{T}_{X}\right)\right)+\sum_{j=1}^{r} \operatorname{dim}_{k} H^{1}\left(G\left(P_{j}\right), \hat{\mathcal{T}}_{X, P_{j}}\right) \\
& =3 g-3+\sum_{j=1}^{r}\left\lceil\frac{d\left(P_{j}\right)}{e_{0}\left(P_{j}\right)}\right\rceil+\sum_{j=1}^{r}\left(\left\lfloor\frac{2 d\left(P_{j}\right)}{e_{0}\left(P_{j}\right)}\right\rfloor-\left\lceil\frac{d\left(P_{j}\right)}{e_{0}\left(P_{j}\right)}\right\rceil\right) \\
& =3 g_{Y}-3+\sum_{j=1}^{r}\left\lfloor\frac{2 d\left(P_{j}\right)}{e_{0}\left(P_{j}\right)}\right\rfloor .
\end{aligned}
$$

The statements (together with some notations) used in the above chain of equalities can be found in [11], [1, pp. 205-206], [10, formula (40)], [1, Proposition 4.1.1]. 
The following lemma gives a different interpretation of the term $\left\lfloor\frac{2 d\left(P_{j}\right)}{e_{0}\left(P_{j}\right)}\right\rfloor$ in Corollary 2.3. To simplify notation we fix one of the points $P_{1}, \ldots, P_{r}$ and write just $P$ for this point and just $d$ and $e_{0}, e_{1}, \ldots$ for $d(P)$ and $e_{0}(P)$, $e_{1}(P), \ldots$, respectively. Furthermore, $N$ and $M$ denote the highest jumps in the lower ramification filtration and in the upper ramification filtration of $G(P)$, respectively.

Lemma 2.5. We have

$$
\left\lfloor\frac{2 d}{e_{0}}\right\rfloor=2(1+M)+\left\lfloor\frac{-2(1+N)}{e_{0}}\right\rfloor .
$$

Proof. Let $k:=\log _{p} e_{0}$. By the Hasse-Arf theorem (see the example on page 76 in [18]) there exist positive integers $a_{0}, a_{1}, \ldots, a_{k-1}$ so that the sequence of jumps in the lower ramification filtration is

$$
i_{1}=a_{o}, \quad i_{2}=a_{0}+p a_{1}, \quad \ldots, \quad i_{k}=a_{0}+p a_{1}+\ldots+p^{k-1} a_{k-1}
$$

and the sequence of jumps in the upper ramification filtration is

$$
a_{0}, \quad a_{0}+a_{1}, \quad \ldots, \quad a_{0}+\ldots+a_{k-1} .
$$

We therefore obtain

$$
\begin{aligned}
d & =\sum_{i=0}^{i_{1}}\left(e_{i_{1}}-1\right)+\sum_{i=i_{1}+1}^{i_{2}}\left(e_{i_{2}}-1\right)+\ldots+\sum_{i=i_{k-1}+1}^{i_{k}}\left(e_{i_{k}}-1\right) \\
& =\sum_{i=0}^{i_{1}}\left(p^{k}-1\right)+\sum_{i=i_{1}+1}^{i_{2}}\left(p^{k-1}-1\right)+\ldots+\sum_{i=i_{k-1}+1}^{i_{k}}(p-1) \\
& =\left(1+i_{1}\right)\left(p^{k}-1\right)+\left(i_{2}-i_{1}\right)\left(p^{k-1}-1\right)+\ldots+\left(i_{k}-i_{k-1}\right)(p-1) \\
& =\left(1+a_{0}\right)\left(p^{k}-1\right)+\left(p a_{1}\right)\left(p^{k-1}-1\right)+\ldots+\left(p^{k-1} a_{k-1}\right)(p-1) \\
& =\left(1+a_{0}+\ldots+a_{k-1}\right) p^{k}-\left(1+a_{0}+p a_{1}+\ldots+p^{k-1} a_{k-1}\right) \\
& =(1+M) p^{k}-(1+N)
\end{aligned}
$$

and hence

$$
\left\lfloor\frac{2 d}{e_{0}}\right\rfloor=\left\lfloor\frac{2(1+M) p^{k}-2(1+N)}{p^{k}}\right\rfloor=2(1+M)+\left\lfloor\frac{-2(1+N)}{e_{0}}\right\rfloor,
$$

as stated. 


\section{The Weakly Ramified Case}

In this section we assume that the cover $\pi: X \rightarrow Y$ is weakly ramified, i.e. that $G_{i}(P)$ is trivial for all $P \in X$ and all $i \geq 2$, and prove the following

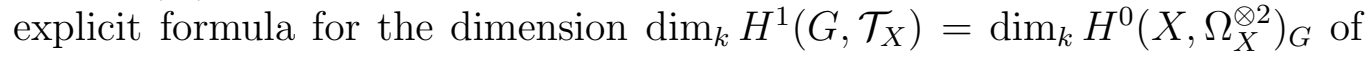
the tangent space of the deformation functor associated with $G$ acting on $X$.

Theorem 3.1. We have

$$
\operatorname{dim}_{k} H^{0}\left(X, \Omega_{X}^{\otimes 2}\right)_{G}=3 g_{Y}-3+\sum_{j=1}^{r} \log _{p}\left|G\left(P_{j}\right)\right|+ \begin{cases}2 r & \text { if } p>3 \\ r & \text { if } p=2 \text { or } 3 .\end{cases}
$$

Remark 3.2. Notice, that if the curve $X$ is ordinary, then the cover $\pi$ is weakly ramified [15, Theorem 2(i)]. In this case, Theorem 3.1 can be proved, similarly to Remark 2.4, using a result of G. Cornelissen and F. Kato on deformations of ordinary curves [4, Theorem 4.5]. In fact, the arguments used in the proof of [4, Theorem 4.5] also work in the weakly ramified case and we thus obtain an alternative proof of Theorem 3.1 in the general case.

Example 3.3. If we moreover assume that $G$ is cyclic then the group $G\left(P_{j}\right)$ is cyclic of order $p$ for all $j=1, \ldots, r$ and the formula in Theorem 3.1 becomes

$$
\operatorname{dim}_{k} H^{0}\left(X, \Omega_{X}^{\otimes 2}\right)_{G}=3 g_{Y}-3+ \begin{cases}3 r & \text { if } p>3 \\ 2 r & \text { if } p=2 \text { or } p=3,\end{cases}
$$

which is the same as in Corollary 2.3 .

Proof (of Theorem 3.1). Let $\Sigma$ denote the skyscraper sheaf defined by the short exact sequence

$$
0 \rightarrow \Omega_{X}^{\otimes 2} \rightarrow \Omega_{X}^{\otimes 2}\left(3 R_{\text {red }}\right) \rightarrow \Sigma \rightarrow 0 .
$$

Since $\operatorname{deg}\left(\Omega_{X}^{\otimes 2}\right)=2\left(2 g_{X}-2\right)>2 g_{X}-2$, we have $H^{1}\left(X, \Omega_{X}^{\otimes 2}\right)=0$ ([7, Example IV 1.3.4]). By applying the functor of global sections we therefore obtain the short exact sequence

$$
0 \rightarrow H^{0}\left(X, \Omega_{X}^{\otimes 2}\right) \rightarrow H^{0}\left(X, \Omega_{X}^{\otimes 2}\left(3 R_{\text {red }}\right)\right) \rightarrow H^{0}(X, \Sigma) \rightarrow 0 .
$$

As the $k[G]$-module $H^{0}\left(X, \Omega_{X}^{\otimes 2}\left(3 R_{\text {red }}\right)\right)$ is projective (by Proposition 3.4 below), its higher group homology vanishes. From the long exact grouphomology sequence associated with the previous short exact sequence we thus obtain the exact sequence

$$
\begin{aligned}
0 \rightarrow H_{1}\left(G, H^{0}(X, \Sigma)\right) \rightarrow & H^{0}\left(X, \Omega_{X}^{\otimes 2}\right)_{G} \\
& \rightarrow H^{0}\left(X, \Omega_{X}^{\otimes 2}\left(3 R_{\mathrm{red}}\right)\right)_{G} \rightarrow H^{0}(X, \Sigma)_{G} \rightarrow 0 .
\end{aligned}
$$


Using a result by the first-named author on the $k[G]$-module structure of Riemann-Roch spaces in the weakly ramified case we will show in Proposition 3.4 below that the $k[G]$-module $H^{0}\left(X, \Omega_{X}^{\otimes 2}\left(3 R_{\text {red }}\right)\right)$ is free and then derive the dimension of $H^{0}\left(X, \Omega_{X}^{\otimes 2}\left(3 R_{\text {red }}\right)\right)_{G}$. Furthermore we will explicitly describe the $k[G]$-module structure of $H^{0}(X, \Sigma)$ and determine (the difference between) the dimension of $H^{0}(X, \Sigma)_{G}$ and of $H_{1}\left(G, H^{0}(X, \Sigma)\right)$ using (unfortunately rather involved) homological computations (see Proposition 3.6 and Lemma 3.7). Theorem 3.1 then immediately follows from the formulas obtained in Propositions 3.4 and 3.6.

Proposition 3.4. The $k[G]$-module $H^{0}\left(X, \Omega_{X}^{\otimes 2}\left(3 R_{\text {red }}\right)\right)$ is a free $k[G]$-module of rank $3\left(g_{Y}-1+r\right)$. In particular we have

$$
\operatorname{dim}_{k} H^{0}\left(X, \Omega_{X}^{\otimes 2}\left(3 R_{\text {red }}\right)\right)_{G}=3\left(g_{Y}-1+r\right) .
$$

Proof. We will first show that the $k[G]$-module $H^{0}\left(X, \Omega_{X}^{\otimes 2}\left(3 R_{\text {red }}\right)\right)$ is free. As $G$ is a $p$-group it suffices to show that it is projective. Let $D=\sum_{P \in X} n_{P}[P]$ be a $G$-invariant divisor on $X$. Theorem 2.1 in [9] tells us that the RiemannRoch space $H^{0}\left(X, \mathcal{O}_{X}(D)\right)$ is projective whenever both $H^{1}\left(X, \mathcal{O}_{X}(D)\right)=0$ and $n_{P} \equiv-1 \bmod e_{1}(P)$ for all $P \in X_{\text {ram }}$. It therefore suffices to check these two conditions for the divisor $D=2 K_{X}+3 R_{\text {red }}$. The first condition follows from [7, Example IV 1.3.4] because $\operatorname{deg}(D) \geq 2\left(2 g_{X}-2\right)>2 g_{X}-2$. The second condition follows from the formulas $K_{X}=\pi^{*} K_{Y}+R$ and

$$
R=\sum_{P \in X_{\text {ram }}} 2\left(e_{0}(P)-1\right)[P] .
$$

Thus $H^{0}\left(X, \Omega_{X}^{\otimes 2}\left(3 R_{\text {red }}\right)\right)$ is a free $k[G]$-module. We now determine its rank. As $H^{1}\left(X, \Omega_{X}^{\otimes 2}\left(3 R_{\text {red }}\right)\right)$ vanishes (see above) we have

$\operatorname{dim}_{k} H^{0}\left(X, \Omega_{X}^{\otimes 2}\left(3 R_{\text {red }}\right)\right)=2\left(2 g_{X}-2\right)+3\left|X_{\text {ram }}\right|+1-g_{X}=3\left(g_{X}-1+\left|X_{\text {ram }}\right|\right)$

by the Riemann-Roch theorem [7, Theorem IV 1.3]. Furthermore the Riemann-Hurwitz formula [7, Corollary IV 2.4] and formula (11) imply that

$$
2 g_{X}-2=|G|\left(2 g_{Y}-2\right)+\sum_{P \in X_{\mathrm{ram}}} 2\left(e_{0}(P)-1\right) .
$$

By combining the previous two equations we obtain

$$
\begin{aligned}
& \operatorname{dim}_{k} H^{0}\left(X, \Omega_{X}^{\otimes 2}\left(3 R_{\text {red }}\right)\right) \\
& =3\left(|G|\left(g_{Y}-1\right)+\sum_{P \in X_{\mathrm{ram}}}\left(e_{0}(P)-1\right)+\left|X_{\mathrm{ram}}\right|\right) \\
& =3|G|\left(g_{Y}-1+r\right) .
\end{aligned}
$$


In particular the rank of $H^{0}\left(X, \Omega_{X}^{\otimes 2}\left(3 R_{\text {red }}\right)\right)$ over $k[G]$ is $3\left(g_{Y}-1+r\right)$ and

$$
\operatorname{dim}_{k}\left(H^{0}\left(X, \Omega_{X}^{\otimes 2}\left(3 R_{\text {red }}\right)\right)_{G}=3\left(g_{Y}-1+r\right),\right.
$$

as stated.

Remark 3.5. A slightly different approach to Proposition 3.4 is to first check the two conditions for the divisor $D=2 K_{X}+3 R_{\text {red }}$ as above but then to use Theorem 4.5 in [9] which computes the isomorphism class of $H^{0}\left(X, \mathcal{O}_{X}(D)\right)$ directly.

Proposition 3.6. If $p>3$ we have

$$
\operatorname{dim}_{k} H_{q}\left(G, H^{0}(X, \Sigma)\right)= \begin{cases}r & \text { for } q=0 \\ \sum_{j=1}^{r} \log _{p}\left|G\left(P_{j}\right)\right| & \text { for } q=1\end{cases}
$$

If $p=3$ we have

$$
\operatorname{dim}_{k} H_{q}\left(G, H^{0}(X, \Sigma)\right)= \begin{cases}r & \text { for } q=0 \\ \sum_{j=1}^{r}\left(\log _{3}\left|G\left(P_{j}\right)\right|-1\right) & \text { for } q=1\end{cases}
$$

If $p=2$ we have

$$
\operatorname{dim}_{k} H_{0}\left(G, H^{0}(X, \Sigma)\right)-\operatorname{dim}_{k} H_{1}\left(G, H^{0}(X, \Sigma)\right)=2 r-\sum_{j=1}^{r} \log _{2}\left|G\left(P_{j}\right)\right| .
$$

Proof. As $\Sigma$ is a skyscraper sheaf, $H^{0}(X, \Sigma)$ is the direct sum of the stalks of $\Sigma$ :

$$
H^{0}(X, \Sigma) \cong \bigoplus_{P \in X_{\mathrm{ram}}} \Sigma_{P} \cong \bigoplus_{j=1}^{r} \operatorname{Ind}_{G\left(P_{j}\right)}^{G}\left(\Sigma_{P_{j}}\right)
$$

We therefore have

$$
H_{q}\left(G, H^{0}(X, \Sigma)\right) \cong \bigoplus_{j=1}^{r} H_{q}\left(G\left(P_{j}\right), \Sigma_{P_{i}}\right) \quad \text { for } q \geq 0
$$

by Shapiro's lemma [23, 6.3.2, p.171]. We now fix one of the points $P_{1}, \ldots, P_{r}$ and write just $P$ for this point. Let $t$ and $s$ be local parameters at $P$ and $\pi(P)$, respectively. Let $m$ denote the multiplicity of a canonical divisor $K_{Y}$ at $\pi(P)$. By formula (1) the multiplicity of the canonical divisor $K_{X}=\pi^{*} K_{Y}+R$ at $P$ 
is then equal to $m e_{0}(P)+2 e_{0}(P)-2$. Hence the multiplicity of $2 K_{X}+3 R_{\text {red }}$ is equal to $2 m e_{0}(P)+4 e_{0}(P)-1$, and we obtain

$$
\Sigma_{P} \cong\left(t^{1-e_{0}(P)(2 m+4)}\right) /\left(t^{4-e_{0}(P)(2 m+4)}\right) \cong(t) /\left(t^{4}\right)
$$

where the latter isomorphism is given by multiplication with the $G(P)$ invariant element $s^{2 m+4}$. We have $g(t) \equiv t \bmod \left(t^{2}\right)$ for all $g \in G(P)$, since $G(P)=G_{1}(P)$. Let the maps $\alpha$ and $\beta$ from $G(P)$ to $k$ be defined by the congruences

$$
g(t) \equiv t+\alpha(g) t^{2}+\beta(g) t^{3} \bmod \quad\left(t^{4}\right)
$$

$g \in G(P)$. The map $\alpha$ is obviously a homomorphism from $G(P)$ to the additive group of $k$ and it is injective because $G_{2}(P)$ is trivial. In particular $G(P)$ is a non-trivial elementary abelian $p$-group. Let $\omega_{1}:=t^{3} \bmod \left(t^{4}\right)$, $\omega_{2}:=t^{2} \bmod \left(t^{4}\right)$ and $\omega_{3}:=t \bmod \left(t^{4}\right)$. The congruences (21) imply that

$$
\begin{aligned}
& g\left(\omega_{1}\right)=\omega_{1} \\
& g\left(\omega_{2}\right)=\omega_{2}+2 \alpha(g) \omega_{1} \\
& g\left(\omega_{3}\right)=\omega_{3}+\alpha(g) \omega_{2}+\beta(g) \omega_{1}
\end{aligned}
$$

for any $g \in G(P)$. Furthermore we easily derive that the map $\beta: G \rightarrow k$ satisfies the condition

$$
\beta(h g)=\beta(h)+2 \alpha(h) \alpha(g)+\beta(g)
$$

for all $h, g \in G(P)$. Now Proposition 3.6 follows from the following homological-algebra lemma which we formulate in a way that is independent from the context of this paper. In the case $p=2$ we need to moreover use Proposition 5.2 which tells us that we can choose the local parameter $t$ in such a way that $\beta=\alpha^{2}$; in particular $\beta$ is not a $k$-multiple of $\alpha$ unless $G(P)$ is cyclic in which case $3-2 \log _{2}|G(P)|=1=2-2 \log _{2}|G(P)|$.

Lemma 3.7. Let $k$ be a field of characteristic $p>0$. Let $G$ be a non-trivial elementary abelian p-group and let $\alpha$ and $\beta$ be maps from $G$ to $k$. We assume that $\alpha$ is a non-zero homomorphism and that $\beta$ satisfies the condition (6) for all $h, g \in G$. Furthermore let $V$ be a vector space over $k$ with basis $\omega_{1}, \omega_{2}, \omega_{3}$. We assume that $G$ acts on $V$ by k-automorphisms given by (3), (4) and (5) (for any $g \in G$ ). If $p>3$, we have

$$
\operatorname{dim}_{k} H_{q}(G, V)= \begin{cases}1 & \text { for } q=0 \\ \log _{p}|G| & \text { for } q=1 .\end{cases}
$$


If $p=3$, we have

$$
\operatorname{dim}_{k} H_{q}(G, V)= \begin{cases}1 & \text { for } q=0 \\ \log _{3}|G|-1 & \text { for } q=1\end{cases}
$$

If $p=2$, we have

$$
\begin{aligned}
\operatorname{dim}_{k} H_{0}(G, V)- & \operatorname{dim}_{k} H_{1}(G, V) \\
& = \begin{cases}3-2 \log _{2}|G| & \text { if } \beta=\text { c } \alpha \text { for some } c \in k, \\
2-\log _{2}|G| & \text { else. }\end{cases}
\end{aligned}
$$

Proof. Let $s$ denote the dimension of $G$ when viewed as vector space over $\mathbb{F}_{p}$, i.e. $s=\log _{p}|G|$, and let $g_{1}, \ldots, g_{s}$ be a basis of $G$ over $\mathbb{F}_{p}$ such that $\alpha\left(g_{1}\right) \neq 0$. For $i=1, \ldots, s$ the sequence

$$
\ldots \stackrel{g_{i}-1}{\longrightarrow} k\left[\left\langle g_{i}\right\rangle\right] \stackrel{1+g_{i}+\ldots+g_{i}^{p-1}}{\longrightarrow} k\left[\left\langle g_{i}\right\rangle\right] \stackrel{g_{i}-1}{\longrightarrow} k\left[\left\langle g_{i}\right\rangle\right] \stackrel{\text { sum }}{\longrightarrow} k \longrightarrow 0
$$

is a $k\left[\left\langle g_{i}\right\rangle\right]$-projective resolution of the trivial $k\left[\left\langle g_{i}\right\rangle\right]$-module $k$, see [23, Section 6.2]. By the Künneth formula [23, Theorem 3.6.3], the tensor product of these sequences is a $k[G]$-projective resolution of the trivial $k[G]$-module $k$ :

$$
\ldots \rightarrow\left(\bigoplus_{i=1}^{s} k[G]\right) \bigoplus\left(\bigoplus_{i<j} k[G]\right) \stackrel{e}{\rightarrow} \bigoplus_{i=1}^{s} k[G] \stackrel{d}{\rightarrow} k[G] \stackrel{\text { sum }}{\rightarrow} k \rightarrow 0
$$

Here the differentials $d$ and $e$ are given as follows: $d$ acts on the $i^{\text {th }}$ direct summand of $\bigoplus_{i=1}^{s} k[G]$ by multiplication with $g_{i}-1$; e maps the $i^{\text {th }}$ direct summand of $\bigoplus_{i=1}^{s} k[G]$ to the $i^{\text {th }}$ direct summand of $\bigoplus_{i=1}^{s} k[G]$ by multiplication with $1+g_{i}+\ldots+g_{i}^{p-1}$ and it maps the direct summand of $\bigoplus_{i<j} k[G]$ indexed by the pair $(i, j)$ to the direct sum of the two direct summands of $\bigoplus_{i=1}^{s} k[G]$ indexed by $i$ and $j$ by multiplication with $g_{j}-1$ and $1-g_{i}$, respectively (note that we have here adopted the iterated sign trick explained in [23, Theorem 3.6.3]). By tensoring the $k[G]$-projective resolution (77) with $V$ over $k[G]$ we obtain the complex

$$
\ldots \rightarrow\left(\bigoplus_{i=1}^{s} V\right) \bigoplus\left(\bigoplus_{i<j} V\right) \rightarrow \bigoplus_{i=1}^{s} V \rightarrow V
$$

which we denote by $C$. (with $V$ sitting in the place 0 ) and whose homology modules are $H .(G, V)$ by definition. We have the filtration

$$
V_{0}:=\{0\} \subset V_{1}:=\operatorname{Span}_{k}\left(\omega_{1}\right) \subset V_{2}:=\operatorname{Span}_{k}\left(\omega_{1}, \omega_{2}\right) \subset V_{3}:=V
$$


of $V$ by $G$-stable subspaces of $V$. By tensoring the $k[G]$-projective resolution (7) of $k$ with $V_{i}$ for $i=0, \ldots, 3$ over $k[G]$ we obtain the filtration

$$
0=F_{0} C . \subset F_{1} C . \subset F_{2} C . \subset F_{3} C .=C .
$$

of the complex $C$. by subcomplexes. Let

$$
E_{\mathbf{p q}}^{1}=H_{\mathbf{p}+\mathbf{q}}\left(F_{\mathbf{p}} C \cdot / F_{\mathbf{p}-\mathbf{1}} C .\right) \Rightarrow H_{\mathbf{p}+\mathbf{q}}(C .)
$$

denote the convergent spectral sequence associated with this filtered complex ([23, Theorem 5.5.1]). (To avoid confusion with the prime $p$ we use $\mathbf{p}$ rather than $p$ to denote the index in the spectral sequence.) Since $G$ acts trivially on $V_{\mathbf{p}} / V_{\mathbf{p}-\mathbf{1}}$ and since the characteristic of $k$ is $p$, both the maps $g_{i}-1$ and $1+g_{i}+\ldots+g_{i}^{p-1}$ act as the zero map on $V_{\mathbf{p}} / V_{\mathbf{p}-\mathbf{1}}$ for all $\mathbf{p}$. Hence all the differentials in $F_{\mathbf{p}} C . / F_{\mathbf{p}-1} C$. are zero and the $E^{1}$-page of our spectral sequence looks as follows (with $V_{1}$ sitting at the place $(1,-1)$ ):

$$
\begin{array}{ccccc}
\vdots & & \vdots & \vdots \\
\bigoplus_{i=1}^{s} V_{1} & \stackrel{\partial_{4}}{\leftarrow} & \left(\bigoplus_{i=1}^{s} V_{2} / V_{1}\right) \bigoplus\left(\bigoplus_{i<j} V_{2} / V_{1}\right) & \leftarrow & \vdots \\
V_{1} & \stackrel{\partial_{3}}{\leftarrow} & \bigoplus_{i=1}^{s} V_{2} / V_{1} & \stackrel{\partial_{2}}{\leftarrow} & \left(\bigoplus_{i=1}^{s} V_{3} / V_{2}\right) \bigoplus\left(\bigoplus_{i<j} V_{3} / V_{2}\right) \\
0 & \leftarrow & V_{2} / V_{1} & \stackrel{\partial_{1}}{\leftarrow} & \bigoplus_{i=1}^{s} V_{3} / V_{2} \\
0 & \leftarrow & 0 & & V_{3} / V_{2}
\end{array}
$$

As all the columns indexed by $p \leq 0$ or $p \geq 4$ are zero we obtain the equalities

$$
\operatorname{dim}_{k} H_{0}(G, V)=\operatorname{dim}_{k} E_{3,-3}^{1}+\operatorname{dim}_{k} E_{2,-2}^{2}+\operatorname{dim}_{k} E_{1,-1}^{3}
$$

and

$$
\operatorname{dim}_{k} H_{1}(G, V)=\operatorname{dim}_{k} E_{3,-2}^{3}+\operatorname{dim}_{k} E_{2,-1}^{2}+\operatorname{dim}_{k} E_{1,0}^{3} .
$$

We now assume that $p>3$ and prove Lemma 3.7 by showing that the six dimensions on the right hand side are equal to $1,0,0, s-1,0$ and 1 , respectively. It is obvious that the (first) dimension $\operatorname{dim}_{k} E_{3,-3}^{1}=\operatorname{dim}_{k} V_{3} / V_{2}$ is equal to 1 . To determine the remaining five dimensions, let $\partial_{1}, \partial_{2}, \partial_{3}$ and $\partial_{4}$ denote the differentials as indicated in the above diagram of the $E^{1}$-page of our spectral sequence. We are now going to explicitly describe 
these differentials by using the fact that they are connecting homomorphisms associated with the short exact sequences of complexes

$$
0 \rightarrow F_{1} C . \rightarrow F_{2} C . \rightarrow F_{2} C . / F_{1} C . \rightarrow 0
$$

and

$$
0 \rightarrow F_{2} C . / F_{1} C . \rightarrow F_{3} C . / F_{1} C . \rightarrow F_{3} C . / F_{2} C . \rightarrow 0 .
$$

For any $a_{1}, \ldots, a_{s} \in k$ we have

$$
\begin{aligned}
\partial_{1} & \left(\left(a_{1} \bar{\omega}_{3}, \ldots, a_{s} \bar{\omega}_{3}\right)\right) \\
\quad & =\overline{\left(g_{1}-1\right)\left(a_{1} \omega_{3}\right)}+\ldots+\overline{\left(g_{s}-1\right)\left(a_{3} \omega_{3}\right)} \\
& =\left(a_{1} \alpha\left(g_{1}\right)+\ldots+a_{r} \alpha\left(g_{s}\right)\right) \bar{\omega}_{2} .
\end{aligned}
$$

In particular, the differential $\partial_{1}$ is surjective (since the homomorphism $\alpha$ is non-zero) and we obtain

$$
\operatorname{dim}_{k} E_{2,-2}^{2}=0
$$

as claimed above, and

$$
\operatorname{dim}_{k} E_{3,-2}^{2}=s-1
$$

Similarly, for any $a_{1}, \ldots, a_{s} \in k$, we have

$$
\partial_{3}\left(\left(a_{1} \bar{\omega}_{2}, \ldots, a_{s} \bar{\omega}_{2}\right)\right)=2\left(\left(a_{1} \alpha\left(g_{1}\right)+\ldots+a_{s} \alpha\left(g_{s}\right)\right) \bar{\omega}_{1} .\right.
$$

In particular $\partial_{3}$ is surjective as well and we obtain

$$
\operatorname{dim}_{k} E_{1,-1}^{3}=\operatorname{dim}_{k} E_{1,-1}^{2}=0,
$$

as claimed above; hence the differential from $E_{3,-2}^{2}$ to $E_{1,-1}^{2}$ is zero and we conclude

$$
\operatorname{dim}_{k} E_{3,-2}^{3}=\operatorname{dim}_{k} E_{3,-2}^{2}=s-1,
$$

as claimed above. As $\alpha\left(g_{1}\right) \neq 0$, the $s-1$ tuples

$$
\begin{aligned}
y_{1} & :=\left(\alpha\left(g_{2}\right) \bar{\omega}_{2},-\alpha\left(g_{1}\right) \bar{\omega}_{2}, 0, \ldots, 0\right) \\
y_{2} & :=\left(\alpha\left(g_{3}\right) \bar{\omega}_{2}, 0,-\alpha\left(g_{1}\right) \bar{\omega}_{2}, 0, \ldots, 0\right) \\
& \vdots \\
y_{s-1} & :=\left(\alpha\left(g_{s}\right) \bar{\omega}_{2}, 0, \ldots, 0,-\alpha\left(g_{1}\right) \bar{\omega}_{2}\right) .
\end{aligned}
$$

of $\bigoplus_{i=1}^{s} V_{2} / V_{1}$ are linearly independent over $k$ and (hence) span the kernel of $\partial_{3}$. For $i=1, \ldots, s-1$ let $x_{i}$ be the tuple of $\left(\bigoplus_{i=1}^{s} V_{3} / V_{2}\right) \bigoplus\left(\bigoplus_{i<j} V_{3} / V_{2}\right)$ 
that has $\bar{\omega}_{3}$ at the place $(1, i+1)$ and 0 everywhere else. From the description of the differential $e$ given above we obtain

$$
\partial_{2}\left(x_{i}\right)=y_{i} \quad \text { for } i=1, \ldots, s-1 .
$$

In particular the image of $\partial_{2}$ is equal to the kernel of $\partial_{3}$ and we conclude

$$
\operatorname{dim}_{k} E_{2,-1}^{2}=0
$$

as claimed above. Similarly we obtain that the dimension of the image of $\partial_{4}$ is $s-1$ and hence

$$
\operatorname{dim}_{k} E_{1,0}^{2}=\operatorname{dim}_{k}\left(\operatorname{coker}\left(\partial_{4}\right)\right)=1 .
$$

To prove that also $\operatorname{dim}_{k} E_{1,0}^{3}=1$ (as claimed above) we will show that the differential

$$
\partial: E_{3,-1}^{2}=\operatorname{ker}\left(\partial_{2}\right) \rightarrow \operatorname{coker}\left(\partial_{4}\right)=E_{1,0}^{2}
$$

is zero. This differential is defined as follows, see [23, Section 5.4]. Let $X \in E_{3,-1}^{2}=\operatorname{ker}\left(\partial_{2}: H_{2}\left(F_{3} C . / F_{2} C.\right) \rightarrow H_{1}\left(F_{2} C . / F_{1} C.\right)\right)$. We write $X$ as the residue class of some $x \in F_{3} C_{2}$. Then $e(x) \equiv e(y) \bmod F_{1} C_{1}$ for some $y \in F_{2} C_{2}$ and $\partial(X)$ is equal to the residue class of $e(x-y)$ in $H_{1}\left(F_{1} C\right.$.). As all the differentials in $F_{2} C . / F_{1} C$. are zero (see above) we may choose $y=0$; in particular $e(x) \in F_{1} C_{1}$ and $\partial(X)$ is equal to the residue class of $e(x) \in$ $\bigoplus_{i=1}^{s} V_{1}$ modulo $\operatorname{im}\left(\partial_{4}\right)$. More precisely, let $x=(\tilde{x}, \hat{x})$ with $\tilde{x} \in \bigoplus_{i=1}^{s} V_{3}$ and $\hat{x} \in \bigoplus_{i<j} V_{3}$. From formula (66) and the fact that $\alpha$ is a homomorphism we obtain

$$
\beta\left(g^{m}\right)=m \beta(g)+\left(\begin{array}{c}
m \\
2
\end{array}\right) 2 \alpha(g)^{2}
$$

for any $g \in G$ and $m \geq 1$. For $i=1, \ldots, s$ we therefore have

$$
\begin{aligned}
& \left(1+g_{i}+\ldots+g_{i}^{p-1}\right)\left(\omega_{3}\right) \\
& \quad=\omega_{3}+\left(\omega_{3}+\alpha\left(g_{i}\right) \omega_{2}+\beta\left(g_{i}\right) \omega_{1}\right)+\ldots+\left(\omega_{3}+\alpha\left(g_{i}^{p-1}\right) \omega_{2}+\beta\left(g_{i}^{p-1}\right) \omega_{1}\right) \\
& \quad=p \omega_{3}+\left(\begin{array}{l}
p \\
2
\end{array}\right) \alpha\left(g_{i}\right) \omega_{2}+\left(\left(\begin{array}{l}
p \\
2
\end{array}\right) \beta\left(g_{i}\right)+\left(\begin{array}{l}
p \\
3
\end{array}\right) 2 \alpha\left(g_{i}\right)^{2}\right) \omega_{1}
\end{aligned}
$$

and hence

$$
\left(1+g_{i}+\ldots+g_{i}^{p-1}\right) \omega_{3}=0
$$

since $\operatorname{char}(k)=p>3$; similarly we have $\left(1+g_{i}+\ldots+g_{i}^{p-1}\right) \omega_{2}=0$ and $\left(1+g_{i}+\ldots+g_{i}^{p-1}\right) \omega_{1}=0$. Therefore $e((\tilde{x}, 0))=0$ and $e(x)=e((0, \hat{x}))$. We may assume that $\hat{x}=\left(c_{i j} \omega_{3}\right)_{i<j}$ with some $c_{i j} \in k$ because $e$ maps any element of $F_{2} C_{3}$ into $\operatorname{im}\left(\partial_{4}\right)$. Then $e((0, \hat{x}))=\left(a_{1} \omega_{2}+b_{1} \omega_{1}, \ldots, a_{s} \omega_{2}+b_{s} \omega_{1}\right)$ 
where $a_{1}, \ldots, a_{s}$ and $b_{1}, \ldots, b_{s}$ are given as follows. Let $M(\alpha)$ denote the matrix whose rows are indexed by $k=1, \ldots, r$, whose columns are indexed by the pairs $(i, j)$ with $i<j$ and whose entry at the place $(k,(i, j))$ is equal to

$$
\begin{cases}\alpha\left(g_{j}\right) & \text { if } k=i \\ -\alpha\left(g_{i}\right) & \text { if } k=j \\ 0 & \text { else. }\end{cases}
$$

Similarly the matrix $M(\beta)$ is defined. Then

$$
\left(\begin{array}{c}
a_{1} \\
\vdots \\
a_{s}
\end{array}\right)=M(\alpha)\left(\left(c_{i j}\right)_{i<j}\right) \quad \text { and } \quad\left(\begin{array}{c}
b_{1} \\
\vdots \\
b_{s}
\end{array}\right)=M(\beta)\left(\left(c_{i j}\right)_{i<j}\right) \text {. }
$$

We know that $a_{1}=\ldots=a_{s}=0$ because $X \in \operatorname{ker}\left(\partial_{2}\right)$. As $\operatorname{im}\left(\partial_{4}\right)$ is equal to the kernel of the map

$$
\bigoplus_{i=1}^{s} V_{1} \rightarrow k, \quad\left(d_{1} \omega_{1}, \ldots, d_{s} \omega_{1}\right) \mapsto \alpha\left(g_{1}\right) d_{1}+\ldots+\alpha\left(g_{s}\right) d_{s}
$$

(see above) we need to show that

$$
\alpha\left(g_{1}\right) b_{1}+\ldots+\alpha\left(g_{s}\right) b_{s}=0 .
$$

For all $i<j$ the $(i, j)$-component of both the vectors

$$
\left(\alpha\left(g_{1}\right), \ldots, \alpha\left(g_{s}\right)\right) M(\beta) \quad \text { and } \quad\left(-\beta\left(g_{1}\right), \ldots,-\beta\left(g_{s}\right)\right) M(\alpha) .
$$

is equal to $\alpha\left(g_{i}\right) \beta\left(g_{j}\right)-\alpha\left(g_{j}\right) \beta\left(g_{i}\right)$. Hence

$$
\left(\alpha\left(g_{1}\right), \ldots, \alpha\left(g_{s}\right)\right) M(\beta)=\left(-\beta\left(g_{1}\right), \ldots,-\beta\left(g_{s}\right)\right) M(\alpha) .
$$

Therefore we have

$$
\begin{aligned}
& \alpha\left(g_{1}\right) b_{1}+\ldots+\alpha\left(g_{s}\right) b_{s}=\left(\alpha\left(g_{1}\right), \ldots, \alpha\left(g_{s}\right)\right)\left(\begin{array}{c}
b_{1} \\
\vdots \\
b_{s}
\end{array}\right) \\
& \quad=\left(\alpha\left(g_{1}\right), \ldots, \alpha\left(g_{s}\right)\right) M(\beta)\left(\left(c_{i j}\right)_{i<j}\right) \\
& \quad=\left(-\beta\left(g_{1}\right), \ldots,-\beta\left(g_{s}\right)\right) M(\alpha)\left(\left(c_{i j}\right)_{i<j}\right) \\
& \quad=\left(-\beta\left(g_{1}\right), \ldots,-\beta\left(g_{s}\right)\right)\left(\begin{array}{c}
a_{1} \\
\vdots \\
a_{s}
\end{array}\right)=\left(-\beta\left(g_{1}\right), \ldots,-\beta\left(g_{s}\right)\right)\left(\begin{array}{c}
0 \\
\vdots \\
0
\end{array}\right)=0,
\end{aligned}
$$


as desired.

We now turn to the case $p=3$. The above proof shows that the first five dimensions are the same as in the case $p>3$. However the (final) dimension $\operatorname{dim}_{k} E_{1,0}^{3}$ is equal to 0 in the case $p=3$, as we are going to prove now. As above we have

$$
\operatorname{dim}_{k} E_{1,0}^{2}=\operatorname{dim}_{k}\left(\operatorname{coker}\left(\partial_{4}\right)\right)=1 .
$$

In order to prove that $\operatorname{dim}_{k} E_{1,0}^{3}=0$ we will show that the differential

$$
\partial: E_{3,-1}^{2}=\operatorname{ker}\left(\partial_{2}\right) \rightarrow \operatorname{coker}\left(\partial_{4}\right)=E_{1,0}^{2}
$$

is surjective. Using the same calculation as in the case $p>3$ we obtain

$$
\left(1+g_{1}+g_{1}^{2}\right)\left(\omega_{3}\right)=2 \alpha\left(g_{1}\right)^{2} \omega_{1} .
$$

Since $\alpha\left(g_{1}\right) \neq 0$, the tuple $\left(2 \alpha\left(g_{1}\right)^{2} \omega_{1}, 0, \ldots, 0\right)$ does not lie in the image of $\partial_{4}$ (use the same reasoning as in the case $p>3$ ). Hence the differential $\partial$ is surjective and we obtain

$$
\operatorname{dim}_{k} E_{1,0}^{3}=0
$$

as claimed above.

We finally prove Lemma 3.7 in the case $p=2$. We may and will assume that not only $\alpha\left(g_{1}\right) \neq 0$ but $\alpha\left(g_{i}\right) \neq 0$ for all $i=1, \ldots, s$ : if $\alpha\left(g_{i}\right)=0$ for some $i>1$, we replace $g_{i}$ by $g_{i} g_{1}$. For brevity, we will write just $\alpha_{i}$ and $\beta_{i}$ for $\alpha\left(g_{i}\right)$ and $\beta\left(g_{i}\right)$, respectively. As $p=2$, the map $\beta$ is a homomorphism as well. Also, the group $G$ acts trivially on both $\omega_{1}$ and $\omega_{2}$ and hence on $V_{2}$. Moreover the norm element $1+g_{i}$ is equal to $g_{i}-1$ and we have

$$
\left(g_{i}-1\right)\left(\omega_{3}\right)=\alpha_{i} \omega_{2}+\beta_{i} \omega_{1}
$$

for all $i=1, \ldots, s$. In particular all differentials in $F_{2} C$. and $C \cdot / F_{2} C$. are zero and the long exact sequence associated with the short exact sequence of complexes

$$
0 \rightarrow F_{2} C . \rightarrow C . \rightarrow C . / F_{2} C . \rightarrow 0
$$

looks as follows:

$$
\cdots \longrightarrow\left(\bigoplus_{i=1}^{s} V_{3} / V_{2}\right) \bigoplus\left(\bigoplus_{i<j} V_{3} / V_{2}\right)
$$

$\partial$

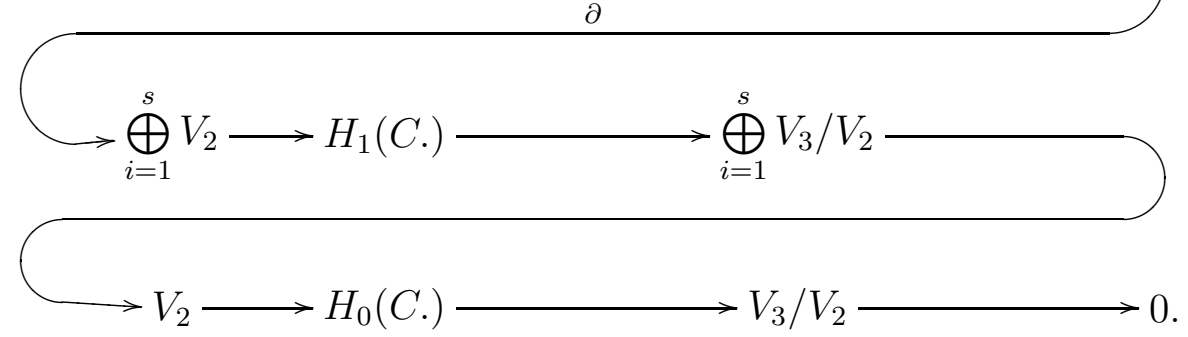


Let $\partial$ denote the connecting homomorphism as indicated above. When restricted to the direct sum $\bigoplus_{i=1}^{s} V_{3} / V_{2}$ the map $\partial$ is the direct sum of the maps $V_{3} / V_{2} \rightarrow V_{2}, a \bar{\omega}_{3} \mapsto a\left(\alpha_{i} \omega_{2}+\beta_{i} \omega_{1}\right), i=1, \ldots, s$. When restricted to the direct summand indexed by the pair $(i, j)$, the map $\partial$ sends the basis element $\bar{\omega}_{3}$ of $V_{3} / V_{2}$ to the tuple of $\bigoplus_{i=1}^{s} V_{2}$ whose $i^{\text {th }}$ component is equal to $\alpha_{j} \omega_{2}+\beta_{j} \omega_{1}$, whose $j^{\text {th }}$ component is equal to $\alpha_{i} \omega_{2}+\beta_{i} \omega_{1}$ and whose other components are all equal to 0 . In particular the dimension of the image of $\partial$ is equal to to rank of the matrix whose columns are indexed by $1, \ldots, s$ and the pairs $(i, j)$ for $i<j$, whose rows are indexed by $1, \ldots, 2 s$ and whose columns indexed by $i$ and $(i, j)$ are equal to

$$
\left(0, \ldots, 0, \alpha_{i}, 0, \ldots, 0 \mid 0, \ldots, 0, \beta_{i}, 0, \ldots, 0\right)^{T}
$$

and

$$
\left(0, \ldots, 0, \alpha_{j}, 0, \ldots, 0, \alpha_{i}, 0, \ldots, 0 \mid 0, \ldots, 0, \beta_{j}, 0, \ldots, 0, \beta_{i}, 0, \ldots, 0\right)^{T},
$$

respectively. Let $R_{1}, \ldots, R_{2 s}$ denote the rows of this matrix. As $\alpha_{1}, \ldots, \alpha_{s}$ are non-zero, the rank does not change if we replace the row $R_{s+i}$ by $\alpha_{i} R_{s+i}+\beta_{i} R_{i}$ for $i=1, \ldots, s$. Then the columns indexed by $i$ and $(i, j)$ are equal to

$$
\left(0, \ldots, 0, \alpha_{i}, 0, \ldots, 0 \mid 0, \ldots, 0\right)^{T}
$$

and

$$
\begin{aligned}
& \left(0, \ldots, 0, \alpha_{j}, 0, \ldots, 0, \alpha_{i}, 0, \ldots, 0 \mid\right. \\
& \left.0, \ldots, 0, \alpha_{i} \beta_{j}+\alpha_{j} \beta_{i}, 0, \ldots, 0, \alpha_{i} \beta_{j}+\alpha_{j} \beta_{i}, 0, \ldots, 0\right)^{T}
\end{aligned}
$$

respectively. In particular the $(s \times s)$-submatrix in the lower left-hand corner is the zero matrix and the $(s \times s)$-submatrix in the upper left-hand corner is the diagonal matrix with diagonal entries $\alpha_{1}, \ldots, \alpha_{s}$ and has rank $s$. If $\beta$ is a $k$-multiple of $\alpha$ the submatrix in the the lower right-hand corner is the zero-matrix as well (or in fact the empty matrix if $s=1$ ) and the rank of the total matrix is $s$. Otherwise, we claim that the rank of the submatrix in the lower right-hand corner is $s-1$, so the rank of the total matrix is $2 s-1$. From the above long exact sequence we then obtain

$$
\begin{aligned}
& \operatorname{dim}_{k} H_{0}(G, V)-\operatorname{dim}_{k} H_{1}(G, V)=\operatorname{dim}_{k} H_{0}(C .)-\operatorname{dim}_{k} H_{1}(C .) \\
& =\operatorname{dim}_{k} V_{3} / V_{2}+\operatorname{dim}_{k} V_{2}-\operatorname{dim}_{k}\left(\bigoplus_{i=1}^{s} V_{3} / V_{2}\right)-\operatorname{dim}_{k} \operatorname{coker}(\partial) \\
& = \begin{cases}1+2-s-(2 s-s)=3-2 s & \text { if } \beta=c \alpha \text { for some } c \in k, \\
1+2-s-(2 s-(2 s-1))=2-s & \text { else, }\end{cases}
\end{aligned}
$$


as claimed above. Finally, to prove the above claim, we first observe that the rank of the submatrix in the lower right-hand corner is at most $s-1$ because the sum of all rows in this submatrix is the zero row. To prove that the rank is at least $s-1$ we will find $s-1$ columns of the submatrix that are linearly independent. To this end, let $i_{1}:=1$; as $\beta$ is not a $k$ multiple of $\alpha$, i.e. as the vector $\left(\beta_{1}, \ldots, \beta_{s}\right)$ is not a $k$-multiple of the vector $\left(\alpha_{1}, \ldots, \alpha_{s}\right)$, there exists $i_{2} \in\{2, \ldots, s\}$ such that $\alpha_{1} \beta_{i_{2}}+\alpha_{i_{2}} \beta_{1} \neq 0$; if $\alpha_{i_{2}} \beta_{i}+\alpha_{i} \beta_{i_{2}}=0$ for all $i \in\{1, \ldots, s\} \backslash\left\{i_{1}, i_{2}\right\}$, let $i_{3}, \ldots, i_{s}$ run through all indices in $\{1, \ldots, s\} \backslash\left\{i_{1}, i_{2}\right\}$; otherwise choose $i_{3} \in\{1, \ldots, s\} \backslash\left\{i_{1}, i_{2}\right\}$ such that $\alpha_{i_{2}} \beta_{i_{3}}+\alpha_{i_{3}} \beta_{i_{2}} \neq 0$; continuing this way we end up with indices $i_{1}, \ldots, i_{s}$ such that $\left\{i_{1}, \ldots, i_{s}\right\}=\{1, \ldots, s\}$ and such that there exists an index $l \in$ $\{1, \ldots, s-1\}$ with the property that $\alpha_{i_{m}} \beta_{i_{m+1}}+\alpha_{i_{m+1}} \beta_{i_{m}}$ is not equal to 0 for $m=1, \ldots, l$ and is equal to 0 for $m=l+1, \ldots, s-1$; in particular we have $\alpha_{i_{l}} \beta_{i_{m}}+\alpha_{i_{m}} \beta_{i_{l}} \neq 0$ as well for $m=l+1, \ldots, s-1$. We now consider the $s-1$ pairs $\widetilde{\left(i_{1}, i_{2}\right)}, \ldots,\left(\widetilde{\left.i_{l-1}, i_{l}\right)}, \widetilde{\left(i_{l}, i_{l+1}\right)}, \ldots, \widetilde{\left(i_{l}, i_{s}\right)}\right.$ where the notation $\widetilde{(i, j)}$ stands for the pair $(i, j)$ if $i<j$ and for $(j, i)$ else. Then the $s-1$ columns $C_{1}, \ldots, C_{s-1}$ corresponding to these $s-1$ pairs are linearly independent: if we have $a_{1}, \ldots, a_{s-1} \in k$ such that $a_{1} C_{1}+\ldots+a_{s-1} C_{s-1}=0$, then by successively looking at the $i_{1}^{\text {th }}, \ldots, i_{l-1}^{\text {th }}$ component we see that $a_{1}=0, \ldots, a_{l-1}=0$ and by finally looking at the $i_{l+1}^{\text {th }}, \ldots, i_{s}^{\text {th }}$ component we see that $a_{l}=0, \ldots, a_{s-1}=0$, as desired.

\section{The $p$-rank representation.}

In this section we assume that $p>3$ and that $\pi$ is not unramified. Let $D$ be a $G$-invariant effective canonical divisor on $X$. We will see in Lemma 4.2 below that such a divisor always exists. As the divisor $D$ is canonical we have an isomorphism between the space

$$
H_{D}:=H^{0}\left(X, \Omega_{X}(D)\right)
$$

and the space $H^{0}\left(X, \Omega_{X}^{\otimes 2}\right)$, this paper's main object of study. As $D$ is effective we furthermore have the decomposition

$$
H_{D}=H_{D}^{\mathrm{s}} \oplus H_{D}^{\mathrm{n}}
$$

where $H_{D}^{\mathrm{s}}$ and $H_{D}^{\mathrm{n}}$ are the spaces of semisimple and nilpotent differentials with respect to the Cartier operator on $H_{D}$, see [13], [20] or [22]. Note that this decomposition depends on the actual divisor $D$ rather than just on its equivalence class; this will become apparent in Theorem 4.3 below, 
for instance. We therefore work with the notation $H_{D}$ rather than with $H^{0}\left(X, \Omega_{X}^{\otimes 2}\right)$ in this section.

Since $D$ is $G$-invariant the above decomposition is a decomposition of $k[G]$ modules. While little seems to be known about the $k[G]$-module $H_{D}^{\mathrm{n}}$, the $k[G]$-module $H_{D}^{\mathrm{s}}$ has been studied by various authors ([2], [13], [20]) and is called the $p$-rank representation. As $G$ is a $p$-group the only irreducible $k[G]$-module is the trivial representation $k$ and has projective cover $k[G][17$, 15.6]. We conclude that

$$
H_{D}^{\mathrm{s}} \cong \operatorname{core}\left(H_{D}^{\mathrm{s}}\right) \oplus k[G]^{b(G, D, k)}
$$

where $\operatorname{core}\left(H_{D}^{\mathrm{s}}\right)$ denotes the direct sum of non-projective indecomposable summands of $H_{D}^{\mathrm{s}}$ (see [20, Definition 2.3]) and $b(G, D, k)$ is called the Borne invariant corresponding to $G, D$ and the trivial representation $k$ (see [20, Definition 5.1]). The goal of this section is to compute the multiplicity $b(G, D, k)$ when we impose further conditions on the divisor $D$, see Theorem 4.3 below. Via the isomorphisms $H_{D} \cong H^{0}\left(X, \Omega^{\otimes 2}\right)$ and $H^{0}\left(X, \Omega^{\otimes 2}\right)_{G} \cong H^{1}\left(G, \mathcal{T}_{X}\right)$, Theorem 4.3 gives us some information on the space $H^{1}\left(G, \mathcal{T}_{X}\right)$, see Corollary 4.4 below, or can be used to derive some information on the nilpotent part $H_{D}^{\mathrm{n}}$ if the $k[G]$-module structure of $H^{0}\left(X, \Omega_{X}^{\otimes 2}\right)$ is known, see Corollary 4.5 ,

We begin with the following lemma which is of independent interest and which we therefore formulate in a way that is independent from the context of this paper.

Lemma 4.1. Let $Z$ be a connected smooth projective curve of genus at least 1 over an algebraically closed field $k$, and let $S$ be a finite set of points on $Z$. Then there exists a global non-zero holomorphic differential on $Z$ such that none of its zeroes belongs to $S$.

Proof. If $g_{Z}=1$ then every non-zero holomorphic differential on $Z$ is nonvanishing [19, III Proposition 1.5], and the result is obvious in this case.

We therefore may and will assume that $g_{Z} \geq 2$. We will prove Lemma 4.1 by induction on $r:=|S|$. The case $r=0$ is trivial. So let $r \geq 1$ and let $P \in S$. For the base step $r=1$ we need to show that

$$
\operatorname{dim}_{k} H^{0}\left(Z, \Omega_{Z}\right)>\operatorname{dim}_{k} H^{0}\left(Z, \Omega_{Z}(-[P])\right) .
$$

By the Riemann-Roch theorem [7, Theorem IV 1.3] the right-hand side is equal to

$$
\left(2 g_{Z}-2-1\right)+1-g_{Z}+l([P])=g_{Z}-2+l([P])=g_{Z}-1
$$


because 1 is a gap number in the sense of the Weierstrass Gap Theorem [21, Theorem I.6.8] since $g_{Z} \geq 2$. Since the left-hand side is equal to $g_{Z}$ this proves the base step $r=1$.

We now prove the inductive step. By the inductive hypothesis there exists a global non-zero holomorphic differential $\omega$ on $Z$ such that none of its zeroes belongs to $S \backslash\{P\}$. Furthermore, by the case $r=1$ there exists a global non-zero holomorphic differential $\phi$ on $Z$ that does not vanish at $P$. So for each $Q \in S$ at least one of the two differentials $\omega, \phi$ does not vanish at $Q$. Hence the sets

$$
\left\{(\lambda, \mu) \in k^{2}: \lambda \omega+\mu \phi \text { vanishes at } Q\right\}, \quad Q \in S,
$$

are one-dimensional subspaces of $k^{2}$. Since $k$ is infinite we can avoid these finitely many lines and hence find a pair $(\lambda, \mu) \in k^{2}$ such that none of the zeroes of $\lambda \omega+\mu \phi$ belongs to $S$.

For the following lemma we recall that the divisor of any non-zero meromorphic differential is a canonical divisor [21, I.5.11].

Lemma 4.2. There exists a $G$-invariant effective canonical divisor $D$ on $X$ whose support contains $X_{\mathrm{ram}}$. Moreover, if $g_{Y}=0$, we may choose $D$ in such a way so that its support is equal to $X_{\text {ram }}$ and, if $g_{Y} \geq 1$, we may choose $D$ of the form $D=\operatorname{div}\left(\pi^{*} \phi\right)$ where $\phi$ is a non-zero holomorphic differential on $Y$ whose zeroes do not belong to $Y_{\text {ram }}$.

Proof. If $\phi$ is a non-zero meromorphic differential on $Y$ then $\pi^{*} \phi$ is a nonzero $G$-invariant meromorphic differential on $X$ and its $\operatorname{divisor} \operatorname{div}\left(\pi^{*} \phi\right)$ is hence a $G$-invariant canonical divisor on $X$. Furthermore we have

$$
\operatorname{div}\left(\pi^{*} \phi\right)=\pi^{*} \operatorname{div}(\phi)+R
$$

by [21, Theorem 3.4.6]. We will choose $\phi$ in such a way so that $D=\operatorname{div}\left(\pi^{*} \phi\right)$ is also effective and its support contains $X_{\text {ram }}$.

If $g_{Y}>0$, then by Lemma 4.1 there exists a non-zero global holomorphic differential $\phi$ on $Y$ whose zeroes do not belong to $Y_{\text {ram }}$. Then $D=\operatorname{div}\left(\pi^{*} \phi\right)$ is certainly effective and its support contains $X_{\text {ram }}$. If $g_{Y}=0$ and $r=1$, we select a generator $x$ of the function field $K(Y)$ of $Y \cong \mathbb{P}_{k}^{1}$ such that $d x=-2\left[\pi\left(P_{1}\right)\right]$ and put $\phi:=d x$. Then we have

$$
\begin{aligned}
& \operatorname{div}\left(\pi^{*} \phi\right)=\pi^{*} \operatorname{div}(\phi)+R \\
& =-2 e_{0}\left(P_{1}\right) \sum_{P \in G P_{1}}[P]+R=\left(-2+\sum_{i=2}^{\infty}\left(e_{i}\left(P_{1}\right)-1\right)\right) \sum_{P \in G P_{1}}[P] .
\end{aligned}
$$


The Riemann-Hurwitz formula

$$
2 g_{X}-2=-2|G|+\sum_{P \in G P_{1}} d\left(P_{1}\right)
$$

[7. Corollary IV 2.4] together with the assumption that $g_{X} \geq 2$ tells us that $\pi$ is not weakly ramified. Hence the coefficient $-2+\sum_{i=2}^{\infty}\left(e_{i}\left(P_{1}\right)-1\right)$ is positive because $p>3$. Thus the divisor $D=\operatorname{div}\left(\pi^{*} \phi\right)$ is effective and its support is equal to $X_{\text {ram }}$, as desired. If $g_{Y}=0$ and $r \geq 2$ we select a generator $x$ of $K(Y)$ such that $x\left(\pi\left(P_{1}\right)\right) \neq \infty$ and $x\left(\pi\left(P_{2}\right)\right) \neq \infty$ and put $\phi=\frac{d x}{\left(x-x\left(\pi\left(P_{1}\right)\right)\right)\left(x-x\left(\pi\left(P_{2}\right)\right)\right)}$. Then we have

$$
\operatorname{div}(\phi)=-\left[\pi\left(P_{1}\right)\right]-\left[\pi\left(P_{2}\right)\right]
$$

and hence

$$
\begin{aligned}
& \operatorname{div}\left(\pi^{*} \phi\right)=-e_{0}\left(P_{1}\right) \sum_{P \in G P_{1}}[P]-e_{0}\left(P_{2}\right) \sum_{P \in G P_{2}}[P]+\sum_{j=1}^{r} \sum_{P \in G P_{j}} d\left(P_{j}\right)[P] \\
& =\sum_{j=1}^{2}\left(-1+\sum_{i=1}^{\infty}\left(e_{i}\left(P_{j}\right)-1\right)\right) \sum_{P \in G P_{j}}[P]+\sum_{j=3}^{r} \sum_{P \in G P_{j}} d\left(P_{j}\right)[P] .
\end{aligned}
$$

Again we see that $D=\operatorname{div}\left(\pi^{*} \phi\right)$ is effective and its support is equal to $X_{\text {ram }}$, as desired.

Let $\gamma_{X}$ and $\gamma_{Y}$ denote the $p$-ranks of $X$ and $Y$, respectively. Furthermore, we recall that $E_{\text {red }}$ denotes the reduced divisor for any effective divisor $E$.

Theorem 4.3. Let $D$ be a $G$-invariant effective canonical divisor on $X$ as in Lemma 4.2. Then the $k[G]$-module $H_{D}^{\mathrm{s}}$ is free of rank

$$
b(G, D, k)= \begin{cases}\gamma_{Y}-1+r & \text { if } g_{Y}=0 \\ \gamma_{Y}-1+r+\operatorname{deg}\left(\operatorname{div}(\phi)_{\mathrm{red}}\right) & \text { if } g_{Y} \geq 1\end{cases}
$$

Proof. By [22, Lemma 2.5], the $k[G]$-module $H_{D}^{\mathrm{s}}$ is the same as $H_{D_{\text {red }}}^{\mathrm{s}}$. Furthermore, if $S$ is any nonempty set of $Y$ containing $Y_{\text {ram }}$ and $E$ is the effective reduced $G$-invariant divisor on $X$ corresponding to $\pi^{-1}(S)$, then the semisimple part of $H^{0}\left(X, \Omega_{X}(E)\right)$ is a free $k[G]$-module of rank $\gamma_{Y}-1+|S|$ by a theorem of Nakajima [13, Theorem 1]. As

$$
D_{\mathrm{red}}= \begin{cases}R_{\mathrm{red}} & \text { if } g_{Y}=0 \\ \operatorname{div}\left(\pi^{*} \phi\right)_{\mathrm{red}}=\left(\pi^{*} \operatorname{div}(\phi)\right)_{\mathrm{red}}+R_{\mathrm{red}} & \text { if } g_{Y} \geq 1\end{cases}
$$


the divisor $D_{\text {red }}$ corresponds to

$$
\begin{cases}X_{\text {ram }}=\pi^{-1}\left(Y_{\text {ram }}\right) & \text { if } g_{Y}=0 \\ \pi^{-1}(\operatorname{supp}(\operatorname{div}(\phi))) \sqcup X_{\text {ram }}=\pi^{-1}\left(\operatorname{supp}(\operatorname{div}(\phi)) \sqcup Y_{\text {ram }}\right) & \text { if } g_{Y} \geq 1\end{cases}
$$

and Theorem 4.3 follows from [13, Theorem 1].

Theorem 4.3 has the following possible applications. Firstly, if $\operatorname{dim}_{k}\left(H_{D}^{\mathrm{n}}\right)_{G}$ and, in the case $g_{Y} \geq 1$, also $\operatorname{deg}\left(\operatorname{div}(\phi)_{\text {red }}\right)$ can be computed, we can also compute the dimension $\operatorname{dim}_{k} H^{1}\left(G, \mathcal{T}_{X}\right)=\operatorname{dim}_{k} H^{0}\left(X, \Omega_{X}^{\otimes 2}\right)_{G}$ of the tangent space of the deformation functor associated with $G$ acting on $X$ :

Corollary 4.4. Let $D$ be as in Theorem 4.3. Then we have

$$
\begin{aligned}
\operatorname{dim}_{k} H^{0}\left(X, \Omega_{X}^{\otimes 2}\right)_{G} \\
\quad= \begin{cases}\operatorname{dim}_{k}\left(H_{D}^{\mathrm{n}}\right)_{G}+\gamma_{Y}-1+r & \text { if } g_{Y}=0 \\
\operatorname{dim}_{k}\left(H_{D}^{\mathrm{n}}\right)_{G}+\gamma_{Y}-1+r+\operatorname{deg}\left(\operatorname{div}(\phi)_{\mathrm{red}}\right) & \text { if } g_{Y} \geq 1 .\end{cases}
\end{aligned}
$$

Proof. Obvious.

For the second application we first introduce the following definition. If a $k[G]$-module $M$ is isomorphic to a direct sum $\bigoplus_{i=1}^{l} M_{i}^{\oplus m_{i}}$ for some pairwise non-isomorphic indecomposable $k[G]$-modules $M_{1}, \ldots, M_{l}$ and some natural numbers $m_{1}, \ldots, m_{l}$, we call $m_{i}$ the multiplicity of $M_{i}$ in $M$. In cases the multiplicity $m_{k[G]}$ of the regular representation $k[G]$ in the $k[G]$-module $H^{0}\left(X, \Omega_{X}^{\otimes 2}\right)$ is known, we can also compute the multiplicity $n_{k[G]}$ of $k[G]$ in the nilpotent part $H_{D}^{\mathrm{n}}$ :

Corollary 4.5. Let $D$ be as in Theorem 4.3. Then we have

$$
n_{k[G]}=m_{k[G]}- \begin{cases}\gamma_{Y}-1+r & \text { if } g_{Y}=0 \\ \gamma_{Y}-1+r+\operatorname{deg}\left(\operatorname{div}(\phi)_{\mathrm{red}}\right) & \text { if } g_{Y} \geq 1\end{cases}
$$

Proof. Obvious.

A formula for $m_{k[G]}$ is known if $G$ is cyclic [3, Theorem 7.23] or if $G$ is elementary abelian [8, Section 3]. For instance, if $G$ is cyclic of order $p$, we can derive from [14, Theorem 1] that

$$
m_{k[G]}=3 g_{Y}-3+\sum_{j=1}^{r}\left\lfloor\frac{\left(N_{j}+2\right)(p-1)}{p}\right\rfloor
$$

where $N_{j}$ is the highest (and single) jump in the lower ramification filtration of $G\left(P_{j}\right)$. 


\section{Appendix}

This appendix gives an account of a structure theorem for finite weakly ramified Galois extensions of local fields of positive characteristic $p$ in the case the Galois group is a $p$-group, see Proposition 5.2 below. This structure theorem is used at the end of the proof of Proposition 3.4 in the main part of this paper, but only in the case $p=2$. It appears as (part of) Proposition 1.4 in 4] and Proposition 1.18 in [5]; in this appendix we give a self-contained and elementary proof. It implies a very explicit and simple description of the action of the Galois group on a local parameter, see Proposition 5.2. In the situation of Section 3 of the main part of this paper we finally derive a certain feature of pole numbers, if $p=2$, see Corollary 5.3.

Let $K$ be a local field of characteristic $p>0$; i.e. the field $K$ is complete with respect to a discrete valuation $v_{K}: K^{\times} \rightarrow \mathbb{Z}$ and its residue field $k$ is perfect; we assume that $k$ is contained in $K$.

Lemma 5.1. Let $L / K$ be a totally ramified Galois extension of degree $p$. Then there exists an element $y \in L$ whose valuation is coprime to $p$ and negative, say $-m$, such that $y^{p}-y \in K$ and $L=K(y)$. The greatest integer $M$ such that the higher ramification group $G_{M}$ of $L / K$ does not vanish is then equal to $m$.

Proof. Let $\sigma$ be a generator of the Galois group $G=\operatorname{Gal}(L / K)$. By the classical Artin-Schreier Theorem there exist elements $x \in K$ and $y \in L$ such that $L=K(y)$ and $y^{p}-y=x$. We first show that the valuation $v_{K}(x)$ of $x$ is negative and that $v_{L}(y)=v_{K}(x)$. Suppose that $v_{K}(x) \geq 0$. Then also $v_{L}(y) \geq 0$ and, denoting the residue classes of $y$ and $x$ in $k$ by $\bar{y}$ and $\bar{x}$, respectively, we obtain

$$
(y-\bar{y})^{p}-(y-\bar{y})=\left(y^{p}-y\right)-\overline{y^{p}-y}=x-\bar{x} .
$$

In particular we have $\sigma(y-\bar{y})=(y-\bar{y})+c$ for some $c \in \mathbb{F}_{p}^{\times}$and

$$
0<v_{L}(y-\bar{y})=v_{L}(\sigma(y-\bar{y}))=v_{L}((y-\bar{y})+c)=0,
$$

which is a contradiction. Hence we have $v_{K}(x)<0, v_{L}(y)<0$ and

$$
p v_{L}(y)=v_{L}\left(y^{p}\right)=v_{L}\left(y^{p}-y\right)=v_{L}(x)=p v_{K}(x),
$$

i.e. $v_{L}(y)=v_{K}(x)$. Let $m:=-v_{L}(y)=-v_{K}(x)$. If $p$ divides $m$, i.e. $m=l p$ for some $l \in \mathbb{N}$, we write

$$
x=\frac{u_{0}}{s^{l p}}+\frac{u_{1}}{s^{l p-1}}+\frac{u_{2}}{s^{l p-2}}+\ldots
$$


with some $u_{0}, u_{1}, u_{2}, \ldots \in k$ and some local parameter $s$ of $K$. Furthermore, as $k$ is perfect, there is a $v_{0} \in k$ such that $v_{0}^{p}=u_{0}$. We now consider

$$
\tilde{y}:=y-\frac{v_{0}}{s^{l}} \in L \quad \text { and } \quad \tilde{x}:=x-\frac{v_{0}^{p}}{s^{l p}}+\frac{v_{0}}{s^{l}} \in K .
$$

Then we have $L=K(\tilde{y})$ and

$$
\tilde{y}^{p}-\tilde{y}=\left(y-\frac{v_{0}}{s^{l}}\right)^{p}-\left(y-\frac{v_{0}}{s^{l}}\right)=\left(y^{p}-y\right)-\frac{v_{0}^{p}}{s^{l p}}+\frac{v_{0}}{s^{l}}=\tilde{x} .
$$

As above we obtain $v_{L}(\tilde{y})=v_{K}(\tilde{x})<0$; furthermore we have

$$
\begin{aligned}
v_{K}(\tilde{x})=v_{K} & \left(x-\frac{v_{0}^{p}}{s^{l p}}+\frac{v_{0}}{s^{l}}\right) \\
& =v_{K}\left(\left(\frac{u_{1}}{s^{l p-1}}+\frac{u_{2}}{s^{l p-2}}+\ldots\right)+\frac{v_{0}}{s^{l}}\right)>-l p=-m=v_{K}(x) .
\end{aligned}
$$

Continuing this way (if necessary) we obtain $x \in K$ and $y \in L$ such $L=$ $K(y), y^{p}-y=x$ and $p$ does not divide $m=-v_{L}(y)=-v_{K}(x)>0$, as claimed.

Let $r, l \in \mathbb{Z}$ such that $r p+l m=1$. Let $s$ be a local parameter of $K$ and put $t:=s^{r} y^{-l} \in L$. Then we have $v_{L}(t)=r p+(-l)(-m)=1$, i.e. $t$ is a local parameter of $L$. Furthermore we have

$$
\sigma(t)=\sigma\left(s^{r} y^{-l}\right)=s^{r} \sigma(y)^{-l}=s^{r}(y-c)^{-l} \text { for some } c \in \mathbb{F}_{p}^{\times},
$$

hence

$$
\sigma(t)=s^{r}\left(\frac{y^{-1}}{1-c y^{-1}}\right)^{l}=t\left(1+l c y^{-1}+\ldots\right)
$$

and finally

$$
v_{L}(\sigma(t)-t)=v_{L}\left(t\left(l c y^{-1}\right)\right)=1+m,
$$

as was to be shown.

Proposition 5.2. Let $L / K$ be a finite weakly ramified Galois extension of local fields such that the residue field $k$ of $K$ is algebraically closed and the Galois group $G$ is a p-group. Then there exist $t_{0} \in K, t_{1}, \ldots, t_{n} \in L$ and $c_{1}, \ldots, c_{n-1} \in k^{\times}$such that $L=K\left(t_{1}, \ldots, t_{n}\right), t:=t_{n}$ is a local parameter of $L, t_{1}^{-p}-t_{1}^{-1}=t_{0}^{-1}$ and $t_{i}^{-p}-t_{i}^{-1}=c_{i-1} t_{i-1}^{-1}$ for $i=2, \ldots, n$. Furthermore for each $g \in G$ there is an $a \in k$ (depending on $g$ ) such that

$$
g(t)=\frac{t}{1-a t}=t\left(1+a t+a^{2} t^{2}+\ldots\right) .
$$


Proof. As $L / K$ is weakly ramified, the Galois group $G$ is in fact an elementary abelian $p$-group, i.e. $G=C_{1} \times \ldots \times C_{n}$ for some subgroups $C_{1}, \ldots, C_{n}$ of $G$ of order $p$. We proceed by induction on $n$. If $n=0$ there is nothing to prove. So let $n \geq 1$. We write just $C$ for $C_{n}$. By the inductive hypothesis there exist $c_{1}, \ldots, c_{n-2} \in k^{\times}, t_{0} \in K$ and $t_{1}, \ldots, t_{n-1}$ in the fixed field $L^{C}$ such that $L^{C}=K\left(t_{1}, \ldots, t_{n-1}\right)$, the element $t_{n-1}$ is a local parameter of $L^{C}$, $t_{1}^{-p}-t_{1}^{-1}=t_{0}$ and $t_{i}^{-p}-t_{i}^{-1}=c_{i-1} t_{i-1}^{-1}$ for $i=2, \ldots, n-1$. By Lemma 5.1 there exist local parameters $s$ and $t$ of $L^{C}$ and $L$, respectively, such that $L=L^{C}(t)$ and $t^{-p}-t^{-1}=s^{-1}$. If $n=1$ we redefine $t_{0}$ to be equal to $s$ and put $t_{1}:=t$. If $n>1$ we write $s^{-1}=c_{n-1} t_{n-1}^{-1}+e$ for some $c_{n-1} \in k^{\times}$and some $e$ in the valuation ring $\mathcal{O}_{L^{C}}$ of $L^{C}$. As $k$ is algebraically closed and $\mathcal{O}_{L^{C}}$ is Henselian we can find $f \in \mathcal{O}_{L^{C}}$ such that $f^{p}-f=e$. We finally define $t_{n}$ to be the inverse of $t^{-1}-f$ and obtain $t_{n}^{-p}-t_{n}^{-1}=\left(t^{-p}-t^{-1}\right)-\left(f^{p}-f\right)=c_{n-1} t_{n-1}^{-1}$, as desired. To prove the last assertion we fix $g \in G$. By the inductive hypothesis there exists $b \in k$ such that $g\left(t_{n-1}\right)=\frac{t_{n-1}}{1-b t_{n-1}}$, i.e. $g\left(t_{n-1}^{-1}\right)=t_{n-1}^{-1}-b$. Then we have

$$
g\left(t^{-1}\right)^{p}-g\left(t^{-1}\right)=g\left(t_{n}^{-p}-t_{n}^{-1}\right)=g\left(c_{n-1} t_{n-1}^{-1}\right)=c_{n-1} t_{n-1}^{-1}-c_{n-1} b .
$$

Hence we have $g\left(t^{-1}\right)=t^{-1}-a$, i.e. $g(t)=\frac{t}{1-a t}$, for some Artin-Schreier root $a$ of $c_{n-1} b$, as desired.

We now return to the situation considered in the main part of this paper. We recall that a natural number $m$ is called a pole number at a point $P \in X$ if there exists a meromorphic function $f$ on $X$ whose pole order at $P$ is $m$ and which is holomorphic everywhere else. The Riemann-Roch theorem (see [7, Theorem IV 1.3]) implies that every integer $m>2 g_{X}-2$ is a pole number, see also [21, Proposition 1.6.6].

Corollary 5.3. Suppose that $p=2$ and that $\pi: X \rightarrow Y$ is weakly ramified. Let $P \in X_{\text {ram }}$ such that $G(P)$ is not cyclic. Then the smallest odd pole number $m$ at $P$ is congruent to 1 modulo 4 and $m-1$ is a pole number as well.

The following proof is a refinement of some arguments given in [12].

Proof. Let $\hat{\mathcal{O}}_{X, P}$ denote the completion of the local ring $\mathcal{O}_{X, P}$ at $P$. Let $t \in \hat{\mathcal{O}}_{X, P}$ be a local parameter at $P$ and let the maps $\alpha$ and $\beta$ from $G(P)$ to $k$ be defined by the congruences

$$
g(t) \equiv t+\alpha(g) t^{2}+\beta(g) t^{3} \bmod \quad\left(t^{4}\right),
$$


$g \in G(P)$. We recall that $\alpha$ is a monomorphism, see the proof of Proposition 3.6. We first observe that changing the local parameter $t$ by multiplication with a unit $u \in \hat{\mathcal{O}}_{X, P}^{\times}$amounts to multiplying $\alpha$ and $\beta$ with $\bar{u}^{-1}$ and $\bar{u}^{-2}$, respectively, where $\bar{u}$ denotes the residue class of $u$ in $k^{\times}$. From Proposition 5.2 we therefore conclude that $\beta=\alpha^{2}$ no matter which local parameter $t$ we choose. We will prove below that we can choose $t$ in such a way that $\beta=\frac{m+1}{2} \alpha^{2}+c \alpha$ for some constant $c \in k$. As $G(P)$ is not cyclic we therefore obtain $\frac{m+1}{2}=1$ in $\mathbb{F}_{2}$ (and $c=0$ ), i.e. $m$ is congruent to 1 modulo 4 , as stated.

Let $f$ be a function in $H^{0}\left(X, \mathcal{O}_{X}(m[P])\right)$ whose pole order at $P$ is $m$. Then the function $f^{-1}$ has a zero at $P$ of order $m$. As $\hat{\mathcal{O}}_{X, P}$ is Henselian and $m$ is odd there exists an $m^{\text {th }}$ root $t$ of $f^{-1}$ in $\hat{\mathcal{O}}_{X, P}$. Obviously, $t$ is a local parameter at $P$. Let $m>m_{1}>\ldots>m_{s}=0$ be the sequence of pole numbers at $P$ smaller than $m$. Note that $m_{1}, \ldots, m_{s}$ are even by assumption. Let $f_{1}, f_{2}, \ldots, f_{s}$ be functions in $H^{0}\left(X, \mathcal{O}_{X}(m[P])\right)$ of pole orders $m_{1}, \ldots, m_{s}$, respectively. Then there exist some units $u_{1}, \ldots, u_{s} \in \hat{\mathcal{O}}_{X, P}^{\times}$such that $f_{1}=\frac{u_{1}}{t^{m_{1}}}, \ldots, f_{s}=\frac{u_{s}}{t^{m_{s}}}$; without loss of generality we may assume that $\bar{u}_{1}=\ldots=\bar{u}_{s}=1$ in $k^{\times}$. As $m[P]$ is a $G(P)$-invariant divisor and $f, f_{1}, \ldots, f_{s}$ form a basis of $H^{0}\left(X, \mathcal{O}_{X}([m P])\right)$ (see [21, p. 34]), there exist maps $c_{0}, \ldots, c_{s}$ from $G(P)$ to $k$ such that

$$
g(f)=c_{0}(g) f+c_{1}(g) f_{1}+\ldots+c_{s}(g) f_{s}
$$

for all $g \in G(P)$; i.e. we have

$$
g\left(\frac{1}{t^{m}}\right)=g(f)=\frac{c_{0}(g)}{t^{m}}+\frac{c_{1}(g) u_{1}}{t^{m_{1}}}+\frac{c_{2}(g) u_{2}}{t^{m_{2}}}+\ldots+\frac{c_{s}(g) u_{s}}{t^{m_{s}}} .
$$

On the other hand, using the geometric series and the binomial theorem we derive the following congruence for each $g \in G(P)$ :

$$
\begin{aligned}
g & \left(\frac{1}{t^{m}}\right)=g(t)^{-m} \\
& \equiv t^{-m}\left(1+\alpha(g) t+\beta(g) t^{2}\right)^{-m} \\
& \equiv t^{-m}\left(1-\alpha(g) t-\beta(g) t^{2}+\alpha^{2}(g) t^{2}\right)^{m} \\
& \equiv t^{-m}\left(1+m\left(-\alpha(g) t-\beta(g) t^{2}+\alpha^{2}(g) t^{2}\right)+\left(\begin{array}{c}
m \\
2
\end{array}\right) \alpha^{2}(g) t^{2}\right) \\
& \equiv \frac{1}{t^{m}}+\frac{-m \alpha(g)}{t^{m-1}}+\frac{-m \beta(g)+\left(\begin{array}{c}
m+1 \\
2
\end{array}\right) \alpha^{2}(g)}{t^{m-2}} \bmod \left(\frac{1}{t^{m-3}}\right) .
\end{aligned}
$$

Let $c \in k$ be defined by the congruence $u_{1} \equiv 1+c t \bmod \left(t^{2}\right)$. By comparing the coefficients in (8) with the coefficients in this latter congruence we 
obtain the following equalities for each $g \in G(P): c_{0}(g)=1 ; m_{1}=m-1$ (because $G(P)$ is non-trivial and $\alpha$ is injective) and $c_{1}(g)=-m \alpha(g) ; c_{1}(g) c=$ $-m \beta(g)+\left(\begin{array}{c}m+1 \\ 2\end{array}\right) \alpha^{2}(g)$ (because $\left.m_{s}<\ldots<m_{2}<m-2\right)$. Now replacing $c_{1}(g)$ with $-m \alpha(g)$ in the latter equation and dividing by $m$ we obtain the desired equations

$$
\beta(g)=\frac{m+1}{2} \alpha^{2}(g)+c \alpha(g), \quad g \in G(P) .
$$

As $m-1=m_{1}$, we have also proved that $m-1$ is a pole number.

\section{References}

[1] José Bertin and Ariane Mézard, Déformations formelles des revêtements sauvagement ramifiés de courbes algébriques, Invent. Math. 141 (2000), no. 1, 195-238.

[2] Niels Borne, A relative Shafarevich theorem, Math. Z. 248 (2004), no. 2, 351-367.

[3] Cohomology of G-sheaves in positive characteristic, Adv. Math. 201 (2006), no. $2,454-515$.

[4] Gunther Cornelissen and Fumiharu Kato, Equivariant deformation of Mumford curves and of ordinary curves in positive characteristic, Duke Math. J. 116 (2003), no. $3,431-470$.

[5] _ Zur Entartung schwach verzweigter Gruppenoperationen auf Kurven, J. Reine Angew. Math. 589 (2005), 201-236.

[6] Hershel M. Farkas and Irwin Kra, Riemann surfaces, Springer-Verlag, New York Berlin, 1980, Graduate Texts in Mathematics, 71.

[7] Robin Hartshorne, Algebraic geometry, Springer-Verlag, New York - Heidelberg, 1977, Graduate Texts in Mathematics, No. 52.

[8] Sotiris Karanikolopoulos, On holomorphic polydifferentials in positive characteristic, arXiv:0905.1196v2 (2010), to appear in Math. Nachr., 25pp.

[9] Bernhard Köck, Galois structure of Zariski cohomology for weakly ramified covers of curves, Amer. J. Math. 126 (2004), no. 5, 1085-1107.

[10] Aristides Kontogeorgis, On the tangent space of the deformation functor of curves with automorphisms, Algebra Number Theory 1 (2007), no. 2, 119-161.

[11] _ Polydifferentials and the deformation functor of curves with automorphisms, J. Pure Appl. Algebra 210 (2007), no. 2, 551-558.

[12] _ The ramification sequence for a fixed point of an automorphism of a curve and the Weierstrass gap sequence, Math. Z. 259 (2008), 471-479.

[13] Shōichi Nakajima, Equivariant form of the Deuring- ̌́afarevič formula for Hasse-Witt invariants, Math. Z. 190 (1985), no. 4, 559-566. 
[14] Action of an automorphism of order $p$ on cohomology groups of an algebraic curve, J. Pure Appl. Algebra 42 (1986), no. 1, 85-94.

[15] _ p-ranks and automorphism groups of algebraic curves, Trans. Amer. Math. Soc. 303 (1987), no. 2, 595-607.

[16] Michael Schlessinger, Functors of Artin rings, Trans. Amer. Math. Soc. 130 (1968), $208-222$.

[17] Jean-Pierre Serre, Linear representations of finite groups, Springer-Verlag, New York - Heidelberg, 1977, Translated from the second French edition by Leonard L. Scott, Graduate Texts in Mathematics, Vol. 42.

[18] _ Local fields, Springer-Verlag, New York - Berlin, 1979, Translated from the French by Marvin Jay Greenberg, Graduate Texts in Mathematics, 67.

[19] Joseph H. Silverman, The arithmetic of elliptic curves, Second Edition, Springer, Dordrecht, 2009, Graduate Texts in Mathematics, vol. 106.

[20] Nicolas Stalder, On p-rank representations, J. Algebra 280 (2004), no. 2, 825-841.

[21] Henning Stichtenoth, Algebraic function fields and codes, Second Edition, SpringerVerlag, Berlin, 2009, Graduate Texts in Mathematics, 254.

[22] Doré Subrao, The p-rank of Artin-Schreier curves, Manuscripta Math. 16 (1975), no. $2,169-193$.

[23] Charles A. Weibel, An introduction to homological algebra, Cambridge University Press, Cambridge, 1994, Cambridge Studies in Advanced Mathematics, 38.

School of Mathematics, University of Southampton, Highfield, Southampton, SO17 1BJ, United Kingdom.

Department of Mathematics, National and Kapodistrian University of Athens, Panepistimioupolis, GR-157 84, Athens Greece. 